\title{
Challenges and advantages in wide- field optical coherence tomography angiography imaging of the human retinal and choroidal vasculature at 1.7-MHz A-scan rate
}

Raju Poddar

Justin V. Migacz

Daniel M. Schwartz

John S. Werner

Iwona Gorczynska 


\title{
Challenges and advantages in wide-field optical coherence tomography angiography imaging of the human retinal and choroidal vasculature at 1.7-MHz A-scan rate
}

\author{
Raju Poddar, ${ }^{a, b, \star}$ Justin V. Migacz, ${ }^{a}$ Daniel M. Schwartz, ${ }^{c}$ John S. Werner, ${ }^{a}$ and Iwona Gorczynska ${ }^{a, d}$ \\ aUniversity of California Davis, Vision Science and Advanced Retinal Imaging Laboratory (VSRI), Department of Ophthalmology and \\ Vision Science, Sacramento, California, United States \\ ${ }^{\mathrm{b} B i r l a}$ Institute of Technology, Department of Bio-Engineering, Mesra, Ranchi, Jharkhand, India \\ 'University of California San Francisco, Department of Ophthalmology and Vision Science, San Francisco, California, United States

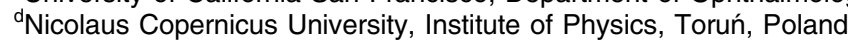

\begin{abstract}
We present noninvasive, three-dimensional, depth-resolved imaging of human retinal and choroidal blood circulation with a swept-source optical coherence tomography (OCT) system at 1065-nm center wavelength. Motion contrast OCT imaging was performed with the phase-variance OCT angiography method. A Fourier-domain mode-locked light source was used to enable an imaging rate of $1.7 \mathrm{MHz}$. We experimentally demonstrate the challenges and advantages of wide-field OCT angiography (OCTA). In the discussion, we consider acquisition time, scanning area, scanning density, and their influence on visualization of selected features of the retinal and choroidal vascular networks. The OCTA imaging was performed with a field of view of 16 deg $(5 \mathrm{~mm} \times 5 \mathrm{~mm})$ and $30 \mathrm{deg}(9 \mathrm{~mm} \times 9 \mathrm{~mm})$. Data were presented in en face projections generated from single volumes and in en face projection mosaics generated from up to 4 datasets. OCTA imaging at $1.7 \mathrm{MHz} \mathrm{A}$-scan rate was compared with results obtained from a commercial OCTA instrument and with conventional ophthalmic diagnostic methods: fundus photography, fluorescein, and indocyanine green angiography. Comparison of images obtained from all methods is demonstrated using the same eye of a healthy volunteer. For example, imaging of retinal pathology is presented in three cases of advanced age-related macular degeneration. $\odot$ The Authors. Published by SPIE under a Creative Commons Attribution 3.0 Unported License. Distribution or reproduction of this work in whole or in part requires full attribution of the original publication, including its DOI. [DOI: 10.1117/1.JBO.22.10.106018]
\end{abstract}

Keywords: optical coherence tomography; retinal blood flow; choroidal blood flow; ophthalmic optics and devices; ophthalmology; medical and biological imaging; optical coherence tomographyangiography; swept-source optical coherence tomography; Fourierdomain mode-locked laser.

Paper 170332RR received May 25, 2017; accepted for publication Oct. 4, 2017; published online Oct. 31, 2017.

\section{Introduction}

The posterior segment of the human eye contains two distinct vascular networks: retinal and choroidal. The retinal vasculature is confined to the inner layers of the retina, from the nerve fiber layer to the outer plexiform layer, and is responsible for blood delivery to and drainage from those layers. The choroidal vasculature is located posterior to the retinal pigment epithelium (RPE) and is isolated from the retina by Bruch's membrane. The function of the choroidal circulation is to provide nutrients to the photoreceptor layer, remove waste materials from the visual cycle, and thermoregulation. Investigation into vascular systems of the eye fundus is important in studies on normal eye function and development of diseases. Many of the ocular diseases leading to blindness, including diabetic retinopathy, ${ }^{1}$ central serous chorioretinopathy, ${ }^{2}$ age-related macular degeneration (AMD) ${ }^{3}$ and glaucoma, ${ }^{4}$ have a vascular component, which manifests as changes in the blood circulation and changes in the vascular morphology. The conventional diagnostic methods used to assess the vascular component of disease development include: fundus photography (FP), fluorescein angiography (FA),

*Address all correspondence to: Raju Poddar, E-mail: rpoddar@bitmesra.ac.in and indocyanine green angiography (ICGA). The typical field of view (FOV) used in these techniques is $30 \mathrm{deg}$ to $45 \mathrm{deg}$, but wide-field fundus cameras can provide an FOV of $\sim 60$ deg. ${ }^{5-7}$ Recently, ultrawide-field imaging systems enabling 200- deg FOV have been developed for FP, FA, and ICGA imaging. 5,6

The advantage of these clinical angiography imaging techniques is that they provide a fast, motion artifact-free, image of the retina. However, they can provide essentially no depth information. FA and ICGA can provide time-resolved analysis of the blood flow distribution. Yet, injection of fluorescent dyes into the blood stream is required, which poses a risk of adverse reactions. Optical coherence tomography angiography (OCTA) imaging techniques can overcome these drawbacks of the classic methods. However, one of the limitations of OCTA is relatively slow imaging speed, making it difficult to capture a wide FOV.

In spectral-domain OCT (SDOCT), the imaging speed is limited by the cameras used in the spectrometers to acquire the OCT signal. Commercially available SDOCT devices operate at $\sim 70-\mathrm{kHz}$ A-scan rate. Systems developed in research laboratories can operate at imaging speeds of $\sim 100$ to $300 \mathrm{kHz}$ with a single camera used for the detection. ${ }^{8}$ The imaging speed can be further increased to over $\sim 0.5 \mathrm{MHz}$ with the use of more than one spectrometer. ${ }^{9,10}$ 
In swept-source OCT (SSOCT) devices, the imaging speed is limited by the sweep rate of the light source. In the commercially available ophthalmic SSOCT systems, the maximum sweep rate is $100 \mathrm{kHz}$. Research grade systems developed for the imaging of the posterior segment of the eye can operate at imaging speeds of $100 \sim 400 \mathrm{kHz}$ with the use of short cavity swept source lasers. ${ }^{11-18}$ Imaging speeds beyond $1 \mathrm{MHz}$ can be achieved with Fourier-domain mode-locked (FDML) lasers. ${ }^{18,19}$ Another technique enabling over 1-MHz imaging is full-field swept source OCT. ${ }^{20}$ Advances in high-speed OCT imaging techniques enabled development of OCT velocimetry (OCT-V) and OCTA methods. OCT-V methods utilize a variety of phenomena to assess some, or all components of the flow velocity vector. These phenomena include: Doppler effect, ${ }^{21}$ Doppler OCT signal broadening, ${ }^{22,23}$ and complex OCT signal decorrelation rate ${ }^{24}$ (measured for example, as complex amplitude, speckle intensity decorrelation or variance, phase decorrelation or variance, or combinations thereof). OCTA ${ }^{25}$ methods use the same phenomena; however, they are not utilized to quantify the flow but are used as intrinsic contrast mechanisms for vascular morphology imaging and quantification. ${ }^{26,27}$

In recent years, many OCTA methods have been developed for the imaging of retinal and choroidal vasculature, including: speckle-variance OCTA, ${ }^{28}$ phase-variance OCTA, ${ }^{29}$ amplitude-decorrelation OCTA (including the split spectrum variant: SSADA), ${ }^{30}$ correlation mapping OCA,${ }^{31}$ optical angiography, ${ }^{32}$ optical microangiography (OMAG), ${ }^{33}$ and power Doppler. $^{34,35}$

Unlike classic, fundus photography, or scanning laser ophthalmosocopy (SLO)-based angiography methods, OCTA techniques provide depth information and enable isolation of all vascular retinal ${ }^{36,37}$ and selected choroidal ${ }^{27,38-41}$ layers. However, imaging speed limitations of the OCT techniques require compromises among a set of imaging parameters. In clinical diagnostics, there is a demand for (1) large FOV, (2) short imaging time, and (3) highly detailed image features. An FOV similar to a standard fundus photograph ( $\sim 30 \mathrm{deg}$ to $45 \mathrm{deg})$ is usually preferred by ophthalmologists to aid in the diagnosis of retinal diseases, but this cannot be achieved with current OCT imaging speeds at high resolution. The rule of thumb for the OCT imaging time is $\leq 5 \mathrm{~s}$ to reduce motion artifacts in the images and to avoid image quality degradation caused by the drying of the tear film. Image quality depends on both the beam spot diameter at the retina and scanning step size. Current speeds of typical OCT devices do not fulfill all three needs for clinical OCTA imaging. If a typical OCT system $(100-\mathrm{kHz}$ A-scan rate, $14-\mu \mathrm{m}$ transverse imaging resolution) is used for OCTA imaging, in which four B-scans are used for the flow metric calculation, then scanning an FOV of $30 \mathrm{deg}(9 \times 9 \mathrm{~mm})$ with a step size of $7 \times 7 \mu \mathrm{m}$ will result in a dataset consisting of $5140 \mathrm{~B}$ scans and 1285 A-scans each. Acquisition time of this dataset would take $1.1 \mathrm{~min}$, which is far beyond acceptable imaging times. If OCTA imaging with an FOV similar to a wide-field fundus angiography is required $(60 \mathrm{deg}, 18 \times 18 \mathrm{~mm})$, the data size would be $10284 \times 2571 \mathrm{~A}$-scans, and the acquisition would take 4.4 min. Clearly, compromise between FOV, scanning step, and imaging time is required in OCTA imaging, depending on specific diagnostic demands. Such compromises have been proposed in previous reports. In the majority of these cases, extending the FOV beyond $10 \mathrm{deg}(3 \mathrm{~mm})$ without exceeding the imaging time beyond 5 to $7 \mathrm{~s}$ was achieved by undersampling in at least one scanning direction. ${ }^{42,43}$ Some authors report reduction in the number of B-scans used for OCTA computations. $^{44,45}$

However, these two approaches may compromise visualization of finer vascular features. In addition, none of these papers reported imaging with an FOV comparable to the classic widefield fundus angiography ( $60 \mathrm{deg})$ in a single dataset. One strategy for achieving a wider FOV in OCTA imaging requires creation of mosaics of several narrow-field images acquired in adjacent locations in the eye fundus. However, this prolongs the patient's imaging session and requires development of data processing methods for overlapping and merging the mosaic tiles. $^{46}$ Some groups have reported wide-field OCTA with realtime motion tracking with a line scan ophthalmoscope for realtime stitching of narrow-field images. ${ }^{42}$ Wide-field OCTA imaging in a single dataset was for the first time reported after the advent of FDML lasers operating at 1060-nm center wavelength and 1.7-MHz imaging speed. ${ }^{38}$

In this paper, we demonstrate OCTA imaging of the posterior eye segment vasculature with an FDML laser operating at 1.7$\mathrm{MHz}$ sweep rate, at 1065-nm center wavelength. The phase-variance method was used for OCT angiography imaging. Images of the retinal and choroidal vasculature with an FOV of 16 deg and 30 deg were captured in single three-dimensional (3-D) datasets. We compare the possibility of imaging selected vascular features depending on the two scan densities. We discuss trade-offs and challenges connected to the imaging speed in the wide-field OCTA, considering acquisition time, scanned area, and scanning density. Imaging was performed in the eyes of a normal subject and three patients diagnosed with AMD.

\section{Materials and Methods}

\subsection{Study Subjects}

Imaging of human subjects was performed under a protocol approved by the UC Davis Institutional Review Board and was in accordance with the declaration of Helsinki. Eyes of a healthy volunteer, 51-year-old Caucasian female (N1), and three patients diagnosed with advanced AMD: geographic atrophy (GA), P1 and P3 ages 60, 81, choroidal neovascularization treated with anti-VEGF injections, $\mathrm{P} 2$, age 67 , was imaged with a swept-source OCT (SSOCT) system. Subject preparation included instillation of eye drops: $1 \%$ tropicamide and $2.5 \%$ phenylephrine, for pupil dilation and cycloplegia. Head position was stabilized with a forehead rest and a bite bar. A white fixation mark was displayed on a liquid crystal display to guide the subject's gaze to select retinal locations for OCT imaging. The light power at the cornea was $1.8 \mathrm{~mW}$, which is below the ANSI limits for safe eye exposure to near-infrared light centered at $1065 \mathrm{~nm}^{47}$

\subsection{Clinical Ophthalmic Imaging}

Standard ophthalmic imaging was performed to enable comparison of features imaged with the 1.7-MHz sweep rate OCTA system and findings visualized using classic diagnostics methods. Color fundus photographs and FA images were taken with a Topcon (TRC-50IX) camera, and ICGA images were collected with the Spectralis HRA + OCT system. In addition, imaging was performed with AngioPlex Cirrus HD-OCT Angiography apparatus (Carl Zeiss Meditec, Inc., California, USA) a spectraldomain OCT (SDOCT) system with 840-nm center wavelength. ${ }^{45}$ The imaging with $5-\mu \mathrm{m}$ axial resolution at full width 
Table 1 Scanning protocols in the Zeiss AngioPlex apparatus..$^{45,48}$

\begin{tabular}{|c|c|c|}
\hline & AngioPlex & AngioPlex \\
\hline Parameter & protocol:1 & protocol: 2 \\
\hline Imaged area & $3 \mathrm{~mm} \times 3 \mathrm{~mm}$ & $6 \mathrm{~mm} \times 6 \mathrm{~mm}$ \\
\hline FOV & 10 deg & 20 deg \\
\hline Pixels per A-scan & 1024 & 1024 \\
\hline Number of A-scans per B-scan & 245 & 350 \\
\hline $\begin{array}{l}\text { Step size (isotropic in horizontal } \\
\text { and vertical direction) }\end{array}$ & $12 \mu \mathrm{m}$ & $17 \mu \mathrm{m}$ \\
\hline Beam spot diameter at the retina & $15 \mu \mathrm{m}$ & $15 \mu \mathrm{m}$ \\
\hline $\begin{array}{l}\text { B-scan repeats (B-scans per } \\
\text { MB-scan) }\end{array}$ & 4 & 2 \\
\hline $\begin{array}{l}\text { Number of BM-scans (slow } \\
\text { scanner positions) }\end{array}$ & 245 & 350 \\
\hline Number of B-scans per volume & 980 & 700 \\
\hline B-scan repetition time & $3.8 \mathrm{~ms}$ & $5 \mathrm{~ms}$ \\
\hline Volume acquisition time & $4 \mathrm{~s}$ & $4 \mathrm{~s}$ \\
\hline
\end{tabular}

Table 2 Axial windowing parameters for en face projection imaging in Zeiss AngioPlex sdOCT apparatus.

\begin{tabular}{ll}
$\begin{array}{l}\text { Imaged vascular } \\
\text { layer }\end{array}$ & Axial span of OCTA en face projections \\
\hline Superficial retina & $\begin{array}{l}\text { From the inner limiting membrane to the outer } \\
\text { boundary of the inner plexiform layer, (layer } \\
\text { segmentation was performed with the Zeiss } \\
\text { AngioPlex software). }\end{array}$ \\
Choriocapillaris & From $\sim 30-$ to $50-\mu \mathrm{m}$ posterior to the RPE peak. \\
Choroid & From $\sim 65-$ to $115-\mu \mathrm{m}$ posterior to the RPE peak.
\end{tabular}

at half maximum (FWHM), and $15-\mu \mathrm{m} 1 / e^{2}$ beam spot diameter at the retina was performed at $68-\mathrm{kHz} \mathrm{A}$-scan rate. The scanning protocols and the parameters of vascular layer visualization are presented in Tables 1 and 2. The OCTA method implemented in the AngioPlex Cirrus HD-OCT instrument is OMAG. All images from the latter system were generated by the AngioPlex software.

\subsection{FDML Swept-Source OCT System}

Imaging was performed with a swept-source OCT system (Fig. 1 and Table 3). A Fourier-domain mode-locked laser (FDML-1060-750-4B-APC, OptoRes GmbH, Germany) was used as the light source with wavelength-sweep frequency of 1.7 MHz, enabling OCT imaging at 1.7 million A-scans/s. The center wavelength of the emitted light was $1065 \mathrm{~nm}$ with $80-\mathrm{nm}$ spectral bandwidth, providing axial imaging resolution of $7 \mu \mathrm{m}$ (FWHM) in tissue. The depth imaging range was $3.5 \mathrm{~mm}$ in tissue, and the peak sensitivity was $92 \mathrm{~dB}$. The imaging platform was designed to provide a maximum FOV of $40 \mathrm{deg}$. A 90:10 fiber coupler (Gooch \& Housego Ltd., UK) was used in the Mach-Zehnder interferometer to split the light between the object arm (10\%) and the reference arm (90\%). The object arm was constructed using

- collimator: two achromatic lenses (Thorlabs, New Jersey, USA), $f=19 \mathrm{~mm}$ each, effective focal length $\sim 11 \mathrm{~mm}$,

- galvanometer scanners (Cambridge Technology, Massachusetts, USA), 3-mm clear aperture,

- scan lens: two achromats (Thorlabs, USA), $f=100 \mathrm{~mm}$ each, effective focal length $\sim 50 \mathrm{~mm}$,

- a dichroic mirror (Chroma Technology, Vermont, USA): transmission of near-infrared light and reflection of visible light, used for coupling of the eye fixation channel with the OCT imaging channel, and

- ocular lens: two achromats (Thorlabs, USA), $f=80 \mathrm{~mm}$ each, effective focal length $\sim 40 \mathrm{~mm}$.

The reference arm consisted of a collimating lens $(f=$ $10 \mathrm{~mm}$, Thorlabs, USA), an iris with adjustable aperture (for controlling the light power), a cube retro-reflector (Thorlabs, USA), and a light-collecting lens ( $f=10 \mathrm{~mm}$, Thorlabs, USA). The light dispersion in the excess of glass in the optical elements of the object arm was compensated with an optical fiber of an experimentally matched length. The polarization control was achieved by a three-paddle polarization controller (Thorlabs,

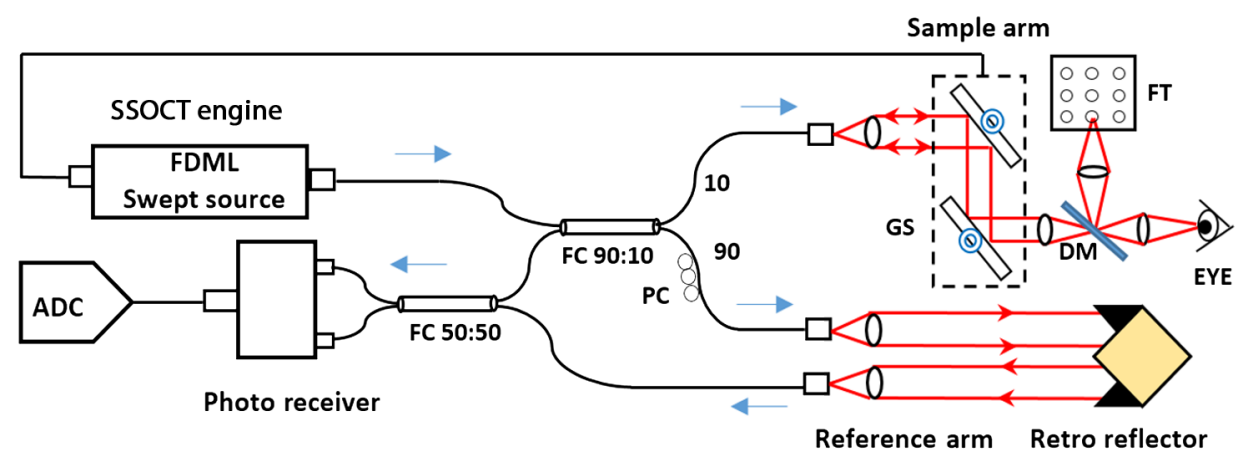

Fig. 1 A diagram of the FDML SSOCT system. FC, fiber coupler; GS, galvanometer; OS, optical scanners; PC, polarization controller; DM, dichroic mirror; FT, fixation target; and ADC, digitizer. 
Table 3 Specifications of the FMDL swept-source OCT system.

\begin{tabular}{lc}
\hline Parameter & Value \\
\hline FDML laser sweep rate & $1.7 \mathrm{MHz}$ \\
FDML laser sweep (A-scan) acquisition time & $0.584 \mu \mathrm{S}$ \\
Central wavelength & $1065 \mathrm{~nm}$ \\
Optical bandwidth (FWHM) & $80 \mathrm{~nm}$ \\
Axial imaging resolution (in tissue, $n \approx 1.33)$ & $7 \mu \mathrm{m}$ \\
$1 / e^{2}$ beam spot diameter at the retina & $14 \mu \mathrm{m}$ \\
Axial imaging range & $3.5 \mathrm{~mm}$ \\
Light power at the cornea & $1.8 \mathrm{~mW}$ \\
Peak imaging sensitivity & $92 \mathrm{~dB}$ \\
\hline
\end{tabular}

USA). The OCT signal was acquired with a dual-balanced detection system containing a 50:50 fiber coupler (Gooch \& Housego Ltd., UK) and a pair of photodiodes (Thorlabs, USA), and digitized using a 12-bit digitizer with sampling speed of 1.8 billion samples/s (ATS9360, Alazar Tech, Canada).

\subsection{OCT Angiography Scanning Protocols Used in FDML SSOCT System}

An overview of the OCT angiography scan protocols used in our FDML SSOCT system is shown in Table 4. Two raster scan protocols with isotropic scanning densities were used: one with a $5-\mu \mathrm{m}$ scan step, and one with an 8- $\mu \mathrm{m}$ scan step. The scanning step in FDML SSOCT protocol 1 was smaller than half of the $1 / e^{2}$ beam spot diameter at the retina $(14 \mu \mathrm{m})$, while the scanning step in FDML SSOCT protocol 2 exceeded that value. Five $\mathrm{B}$-scans were acquired at each position of the slow scanner (per each BM-scan) to enable OCT angiography imaging. A large number of over $1000 \mathrm{~A}$-scans per B-scan and BM-scans per volume were acquired to image the largest possible area of the retina in a time of 6 to $8 \mathrm{~s}$. The data sizes were 11 and $14 \mathrm{~GB}$.

\subsection{Data Processing}

Data processing consisted of three steps: (i) processing of the acquired spectral interference signals, (ii) OCT angiography computations, and (iii) implementation of 3-D data visualization methods for en face projection imaging.

Numerical processing of the OCT signal included generation of the spectral interference pattern in wave number space, from the signal acquired in the wavelength domain, with the use of calibration fringe pattern (often called a k-clock) acquired prior to the patient imaging sessions. Fixed pattern noise was estimated by averaging of all acquired interference spectra, and removed by subtracting the computed mean interference spectrum from each B-scan. Numerical compensation of the residual dispersion mismatch of light propagating in the reference and object arms of the interferometer was performed to avoid axial imaging resolution degradation. Spectral shaping was implemented to reduce image artifacts introduced by the side lobes of the coherence function (OCT axial point-spread function). Finally, digital Fourier transformations with zero-padding to
Table 4 FDML SSOCT angiography scan protocols.

\begin{tabular}{|c|c|c|}
\hline \multirow[b]{3}{*}{ Parameter } & FDML & FDML \\
\hline & SSOCT & SSOCT \\
\hline & Protocol: 1 & protocol: 2 \\
\hline Imaged area & $5 \mathrm{~mm} \times 5 \mathrm{~mm}$ & $9 \mathrm{~mm} \times 9 \mathrm{~mm}$ \\
\hline FOV & 16 deg & $30 \mathrm{deg}$ \\
\hline Pixels per A-scan & 1024 & 1024 \\
\hline Number of A-scans per B-scan & 1024 & 1152 \\
\hline Step size (horizontal and vertical) & $5 \mu \mathrm{m}$ & $8 \mu \mathrm{m}$ \\
\hline $\begin{array}{l}1 / e^{2} \text { beam spot diameter at } \\
\text { the retina }\end{array}$ & $14 \mu \mathrm{m}$ & $14 \mu \mathrm{m}$ \\
\hline $\begin{array}{l}\text { B-scan repeats (B-scans per } \\
\text { BM-scan) }\end{array}$ & 5 & 5 \\
\hline $\begin{array}{l}\text { Number of BM-scans (slow } \\
\text { scanner positions) }\end{array}$ & 1024 & 1152 \\
\hline Number of B-scans per volume & 5120 & 5760 \\
\hline $\begin{array}{l}\text { B-scan repetition time (including } \\
\text { fly-back) }\end{array}$ & $1.2 \mathrm{~ms}$ & $1.35 \mathrm{~ms}$ \\
\hline Volume acquisition time & $6 s$ & $8 \mathrm{~s}$ \\
\hline Data size & $11 \mathrm{~GB}$ & $14 \mathrm{~GB}$ \\
\hline
\end{tabular}

4096 points per spectrum was performed yielding complex amplitudes corresponding to OCT A-scans.

For angiogram generation, we have selected the phase-variance method (PV OCTA) ${ }^{29}$ Magnitudes and phases of the interference fringe pattern at each imaged depth were extracted from the complex amplitudes. The magnitudes were used for intensity thresholding performed to exclude noise pixels from phase-variance computations, and for generation of mean OCT intensity cross-sectional images revealing the tissue morphology. Mean B-scans were computed by averaging B-scans within BMscans. The phases were used to generate cross-sectional OCTA images of the vascular morphology. The OCTA computations included: removal of the phase shifts caused by bulk eye motion (a shifted histogram analysis method was used ${ }^{25}$ ), and computation of phase-variances among B-scans within BM-scans. High values of the phase-variance indicate the location of the moving blood cells within the tissue volume. Projections of the blood flow in the retinal vessels onto deeper layers (blood flowprojection $^{49}$ artifacts $^{37}$ ) were not removed.

Flattening of the B-scans and OCTA cross-sections was performed using methods described in a previous paper. ${ }^{36}$ The image processing included: segmentation of the RPE in the intensity B-scans, flattening the cross-sectional images to the RPE [Fig. 2(b)] using Zernike polynomial fits, removal of the bulk motion affected B-scans from the 3-D datasets. En face projections were generated with ImageJ software ${ }^{50}$ by axial summation of the data within square windows at selected depth locations relative to the RPE position (Table 5). To remove the residual noise from the en face OCT angiography images, 


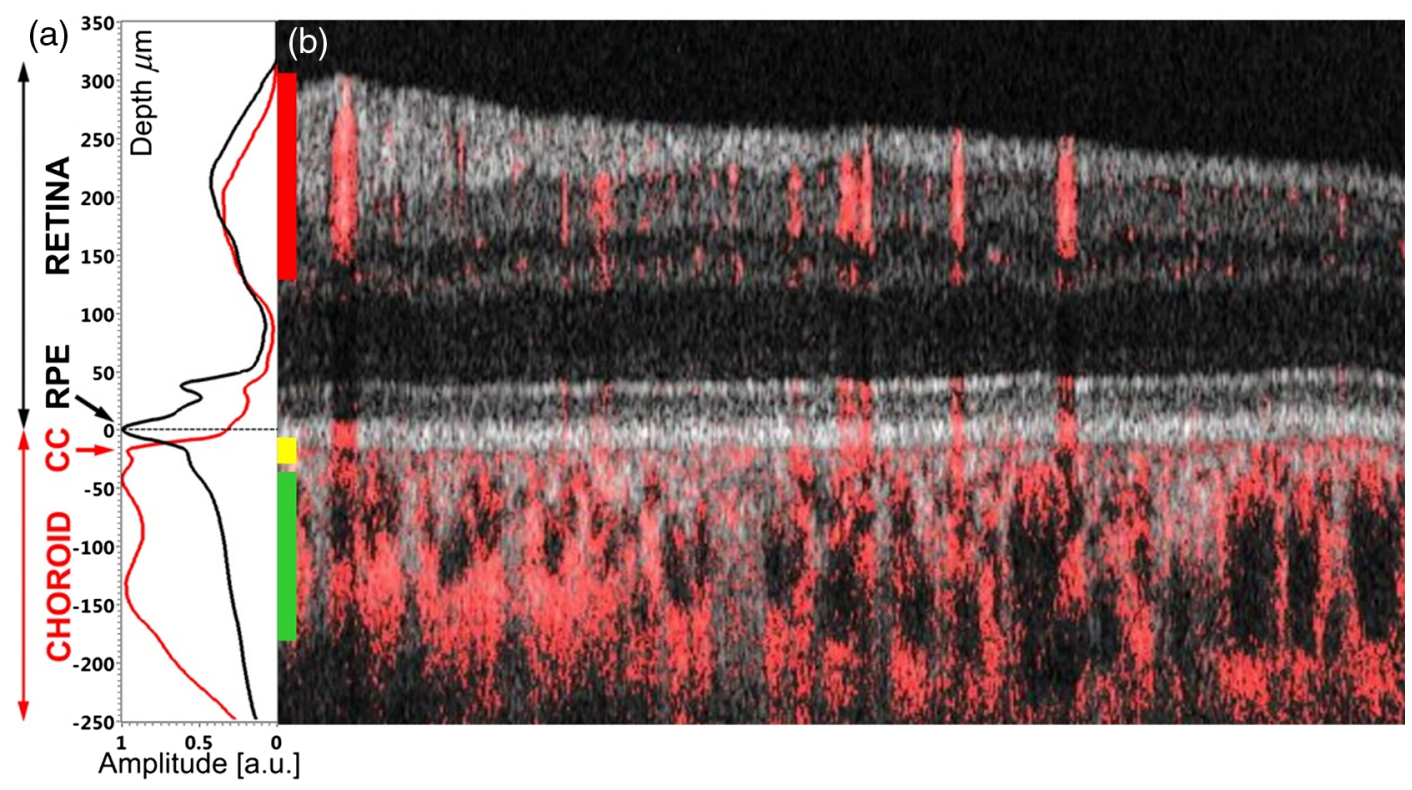

Fig. 2 Example result of cross-sectional images flattened to the RPE obtained in a healthy subject (N1). (a) Mean axial PV OCTA profile (red line) and intensity profile (black line) created by summation of all OCTA and OCT A-scans within the 3-D dataset, CC, choriocapillaris; RPE, retinal pigment epithelium. (b) A composite cross-sectional image generated by an overlay of PV OCTA B-scan (red) with an intensity B-scan (grayscale). Vertical bars in (b) indicate locations and thicknesses of layers selected for the visualization of retinal (red bar), and choroidal (yellow and green bars) vasculature. Horizontal image size: $5 \mathrm{~mm}$.

median filtering was applied followed by a Gaussian filter. Image filtering was performed with ImageJ.

\section{Results}

\subsection{Influence of Scan Density on OCTA Imaging}

The effects of scanning density on the OCT angiography visualization of vascular features were shown in Figs. 3-8. Figures 3,5, and 7 present images obtained from the researchgrade FDML SSOCT system. For comparison with current standards in clinical OCTA imaging, we have also provided angiograms generated with a commercial OCT angiography system (Figs. 4, 6, and 8). For consistency of the results (i.e., to avoid confounding factors caused by inter-subject variability), we have performed imaging using both systems in the same eye of a healthy volunteer (Caucasian female, age: 51 years).

OCT angiography imaging with the FDML SSOCT system provides visualization of not only the retinal (Fig. 3), but also choroidal vasculature (Figs. 5 and 7). Images obtained from the

Table 5 Axial windowing parameters for en face projection imaging in FDML SSOCT system.

\begin{tabular}{llc} 
Imaged vascular layer & \multicolumn{1}{c}{ Window location } & Window width \\
\hline Joint retinal layers & $\begin{array}{l}\sim 90 \mu \mathrm{m} \text {, anterior to } \\
\text { RPE peak }\end{array}$ & $\sim 138 \mu \mathrm{m}$ \\
Choroid 1 (CHRD1) & $\begin{array}{l}\sim 12 \mu \mathrm{m} \text {, posterior to } \\
\text { RPE peak }\end{array}$ & $\sim 28$ to $39 \mu \mathrm{m}$ \\
Choroid 2 (CHRD2) & $\begin{array}{l}\sim 127 \mu \mathrm{m}, \text { posterior to } \\
\text { RPE peak }\end{array}$ & $\sim 120$ to $180 \mu \mathrm{m}$ \\
\hline
\end{tabular}

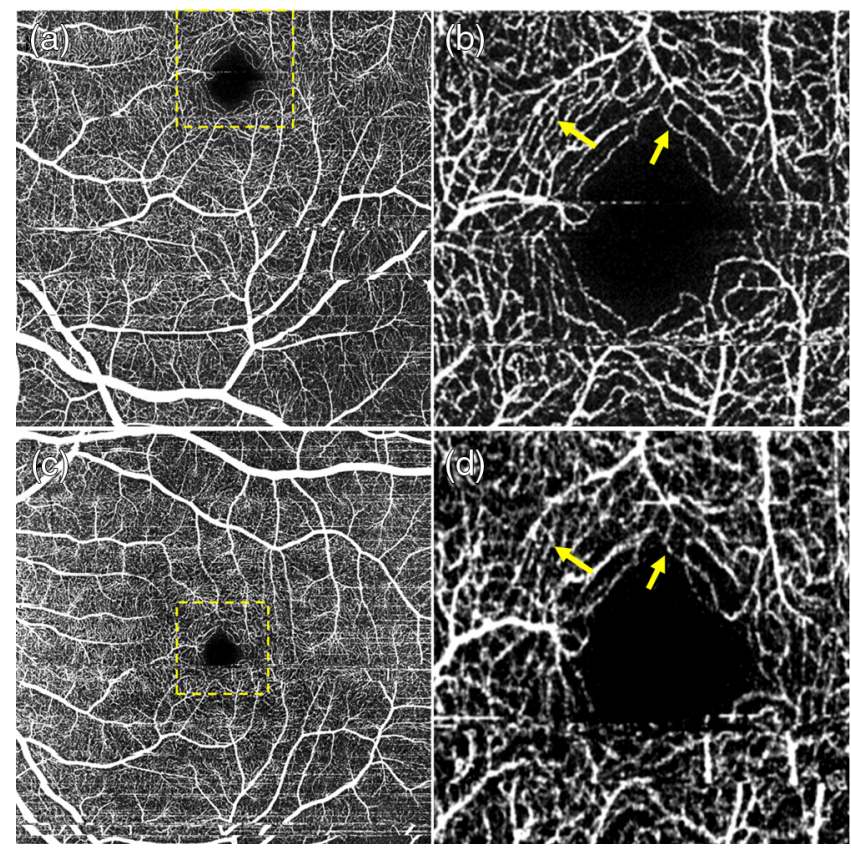

Fig. 3 FDML SSOCT at 1.7-MHz A-scan rate, PV OCTA en face projections of the retinal vasculature. Normal subject, age 51. Top row: scanning step of $5 \mu \mathrm{m}$. Bottom row: scanning step of $8 \mu \mathrm{m}$. Left column: full imaged area. Right column: zoom at $1.5 \mathrm{~mm} \times 1.5 \mathrm{~mm}$ area centered at the fovea. (a) $1024 \times 1024$ pixels, 5-mm $\times 5$-mm area imaged in $6 \mathrm{~s}$. (c) $1152 \times 1152$ pixels, $9-\mathrm{mm} \times 9-\mathrm{mm}$ area imaged in $8 \mathrm{~s}$. Depth positions and ranges used for generating the en face images are given in Table 3. Dashed yellow square shows the zoomed image portion. (b) Arrows indicate example vessels with more continuous appearance and (d) less clearly defined. 


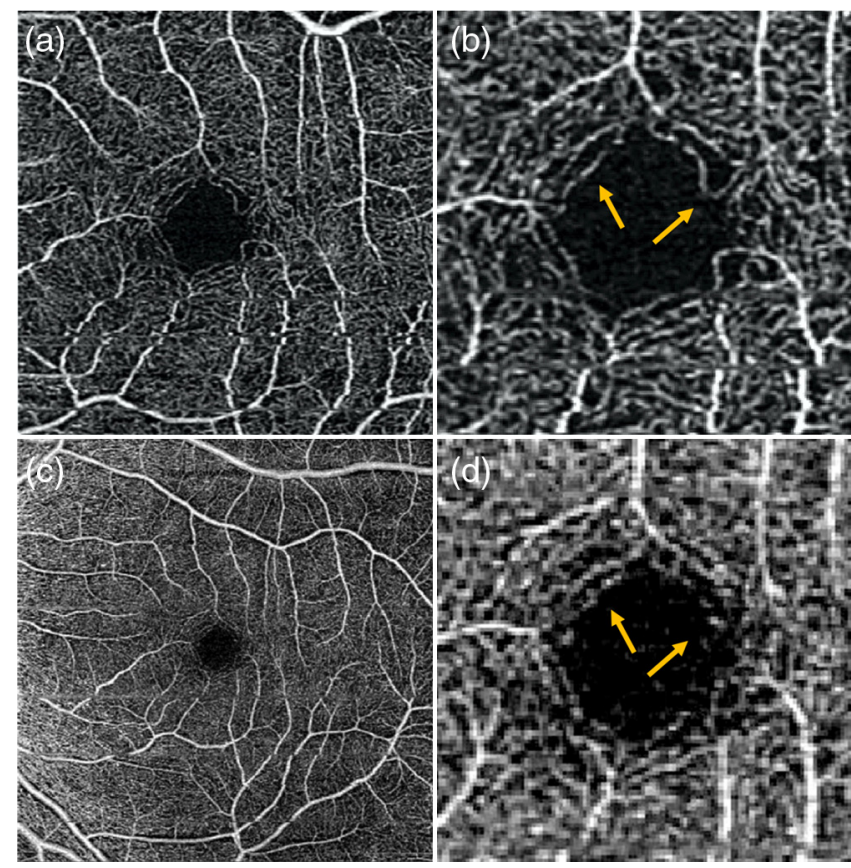

Fig. 4 Zeiss Angioplex 68-kHz A-scan rate, OCTA en face projections of the superficial retina. Normal subject (N1), age 51. Top row: scanning step $12 \mu \mathrm{m}$. Bottom row: scanning step $17 \mu \mathrm{m}$. Left row: full imaged area. Left row: zoom at $1.5-\mathrm{mm} \times 1.5-\mathrm{mm}$ area centered at the fovea. Image sizes: (a) $3 \mathrm{~mm} \times 3 \mathrm{~mm}$, (c) $6 \mathrm{~mm} \times 6 \mathrm{~mm}$, and (b and d) $1.5 \mathrm{~mm} \times 1.5 \mathrm{~mm}$.

commercial OCT angiography apparatus provide images of the retinal vasculature (Fig. 4), but the details of the choroid are not visualized (Fig. 8). However, the reader should be made aware of the differences between the two imaging systems. The research instrument is a swept source OCT system operating at 1.7-MHz A-scan rate, with 1065-nm center wavelength of the light used for the imaging, and uses the PV OCT angiography method. The commercial apparatus uses the spectral-domain OCT technique, with a $68 \mathrm{kHz}$ imaging rate and 840-nm center wavelength of the light emitted by a superluminescent diode and uses the OMAG OCTA method. The two OCT technologies, imaging speeds, and wavelength regimes may influence the possibility to image the choroid and detect the blood flow in the choroidal vessels. ${ }^{51}$

Therefore, the comparison of the images should focus on the details of the visualized vascular features depending on the scanning density. No attempt should be made to identify specific imaging parameters responsible for the lack of choroidal vasculature visualization in the commercial apparatus since our experiments were not designed to support such study.

Figure 3 shows en face projections of the retinal vasculature obtained from the FDML SSOCT system. Vessels arching around the fovea, and branching into vessels radiating toward/ from the fovea are visible in images obtained from the 5- and $8-\mu \mathrm{m}$ scan steps ( 0.36 and 0.57 of the $1 / e^{2}$ beam spot diameter, respectively).

The image area between the large retinal vessels is occupied by a fine meshwork of capillary vessels, located between the inner and outer plexiform layers, or the intermediate and deep plexuses. Visualization of large retinal vessels is not influenced by the implemented scan densities [Figs. 3(a) and 3(c)]. Comparable levels of detail are also visible in the capillary network [Figs. 3(b) and 3(d)]. However, the apparent continuity of
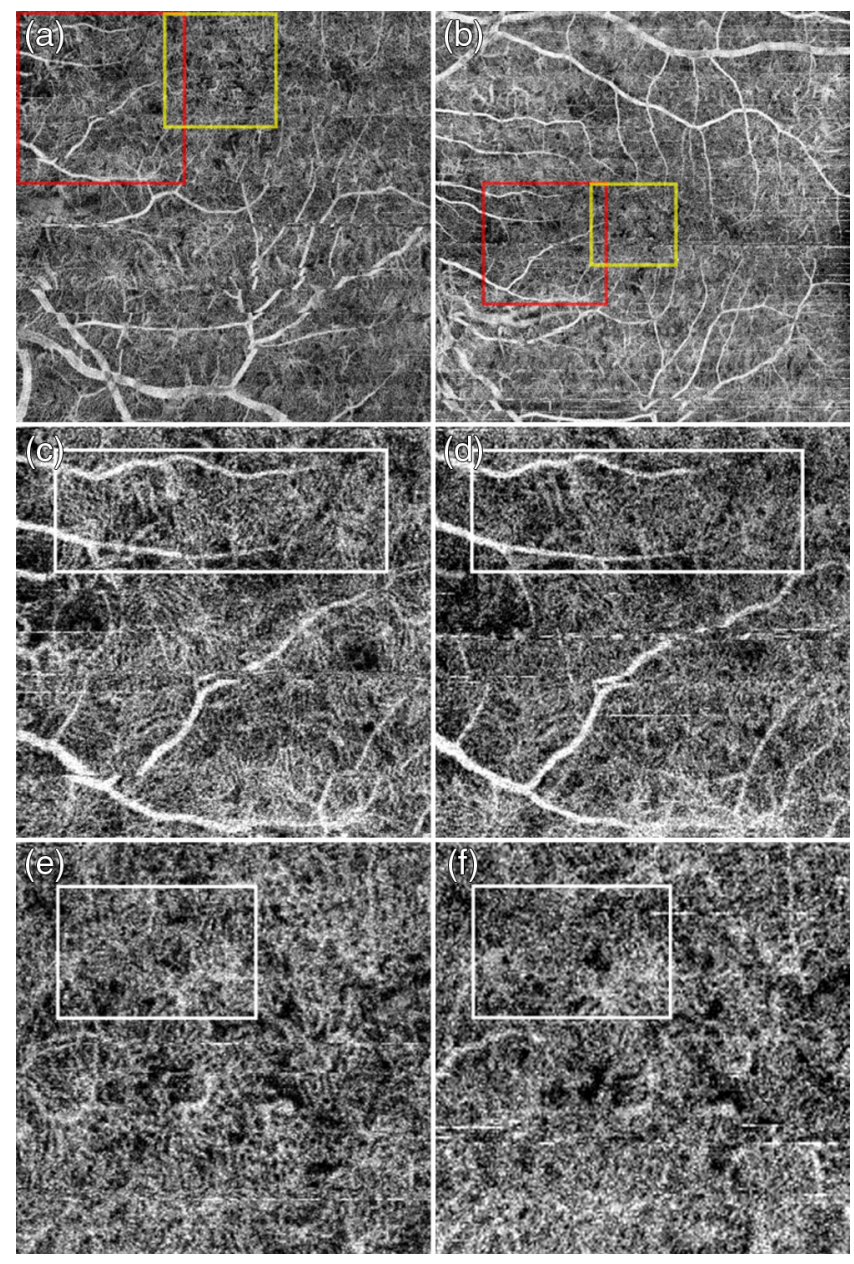

Fig. 5 FDML SSOCT at 1.7-MHz A-scan rate, PV OCTA en face projections of the choriocapillaris. Normal subject, age 51. Left column: scanning step $5 \mu \mathrm{m}$. Right column: scanning step $8 \mu \mathrm{m}$. Top row: full imaged area. Middle row: zoom at 2- $\mathrm{mm} \times 2-\mathrm{mm}$ area indicated by red square in (a) and (b). Bottom row: zoom at 1.5-mm $\times 1.5-\mathrm{mm}$ area centered at the fovea as indicated by yellow square in (a) and (b). Image sizes: (a) $5 \mathrm{~mm} \times 5 \mathrm{~mm}$, (b) $9 \mathrm{~mm} \times 9 \mathrm{~mm}$, (c and d) $2 \mathrm{~mm} \times 2 \mathrm{~mm}$, and (b and d) $1.5 \mathrm{~mm} \times 1.5 \mathrm{~mm}$. Depth position and range selected for generation of the en face images are given in Table 3. (c and e) White rectangles indicate region of interest with appearance resembling the meshwork of choriocapillaris vessels and ( $d$ and $f$ ) less clear visualization of the meshwork due to higher pixilation noise.

vessels in the areas free from saccadic motion artifacts is higher in the images obtained from higher scanning densities of the retinal vasculature, typical for OCTA imaging with commercially available instruments are shown in Fig. 4. Good visualization of large retinal vessels was obtained from the 12- and $17-\mu \mathrm{m}$ scan steps ( 0.8 and 1.1 of the beam spot diameter, respectively). However, visualization of capillary vessels differs for the two scanning densities. While the $12-\mu \mathrm{m}$ scan step provides visualization comparable with the FDML SSOCT system at lower scanning density, the $17-\mu \mathrm{m}$ scan step causes loss of information. Discontinuities are visible in the ring of capillary vessels surrounding the foveal avascular zone [arrows in Figs. 4(b) and 4(d)]. Pixilation noise degrades the quality of the images.

En face projections of the "choriocapillais" layer generated with the FDML SSOCT system are presented in Fig. 5. The enlarged portions of the images obtained from the 5- $\mu \mathrm{m}$ scanning step size (less than half of the $1 / e^{2}$ beam spot diameter) 

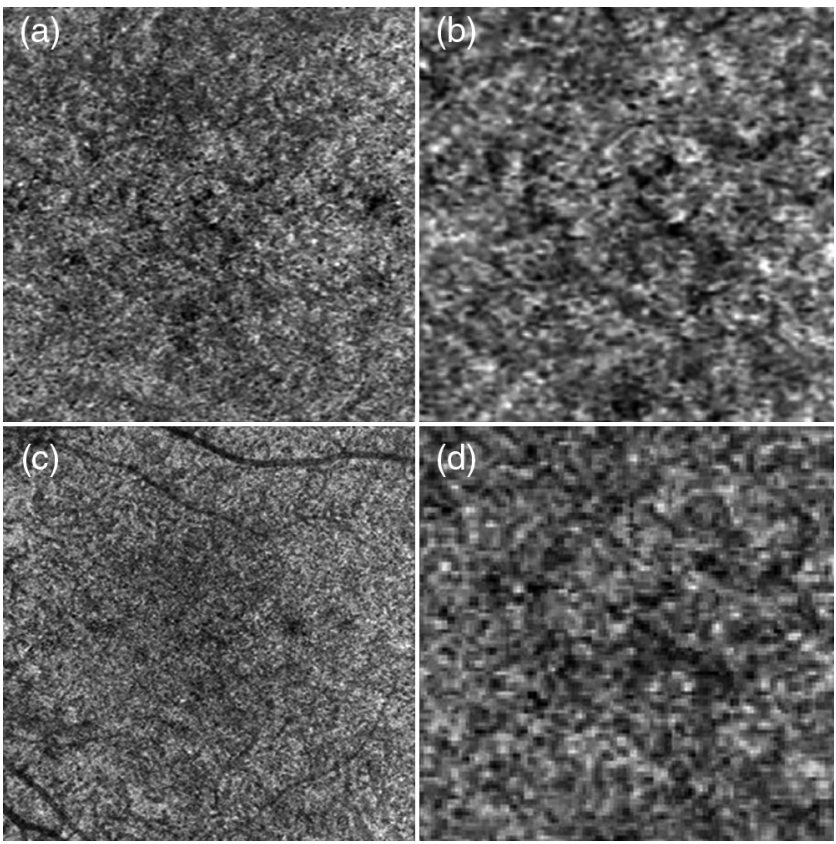

Fig. 6 Zeiss Angioplex, 68-kHz A-scan rate, OCTA en face projections of the choriocapillaris. Normal subject, age 51. Top row: scanning step $12 \mu \mathrm{m}$. Bottom row: scanning step $17 \mu \mathrm{m}$. Left row: full imaged area. Left row: zoom at $1.5-\mathrm{mm} \times 1.5-\mathrm{mm}$ area centered at the fovea. Image sizes: (a) $3 \mathrm{~mm} \times 3 \mathrm{~mm}$, (c) $6 \mathrm{~mm} \times 6 \mathrm{~mm}$, and (b and d) $1.5 \mathrm{~mm} \times 1.5 \mathrm{~mm}^{2}$.

have an appearance resembling a meshwork of choriocapillaris. The meshwork is less evident in the images obtained from $8-\mu \mathrm{m}$ scanning step due to higher pixilation noise. Example regions of interest minimally affected by motion artifacts are indicated by white rectangles. The images of deeper choroid (Fig. 7) show an extremely densely packed network of medium and large diameter vessels. As in OCTA imaging of the large retinal vessels, the visualization of large choroidal vessels is not affected by the two implemented scanning protocols. The appearance of the choroidal vessels in OCTA imaging depends on the size of the vessels and the blood flow velocity. In vessels with smaller lumen and slower flow, the OCTA signal is stronger, most likely due to less washout of the interference fringe pattern and due to less light scattering. These vessels appear white in the en face projections. In the vessels with faster blood flow and with larger lumen, the OCTA signal is weaker or indistinguishable from the noise making their appearance dark in the projection images. However, in most of the large vessels, the OCTA signal is still visible in their interior parts (on the top in the cross-sectional images). If the en face projection includes the top part of a large vessel, that vessel also appears white in the image. In addition, when the en face projection spans in depth below the vessels, the flow projections contribute to the OCTA signal enhancing the visualization.

The presence of the layer, but not the structure, of choriocapillaris was visualized with the commercial OCTA apparatus (Fig. 6). No OCTA signal was detected in deeper choroidal vessels (Fig. 8). Therefore, no reliable conclusions regarding influence of scan density on vascular imaging can be made. However, a few interesting features may be observed in the images of choriocapillaris and deep choroid. In Fig. 6, focal areas with no flow signal (black spots) are embedded within an apparently random distribution of the OCTA signal originating from unresolved choriocapillaris meshwork. The nonperfused spots probably correspond to larger gaps between the vessels (flow voids). ${ }^{52}$

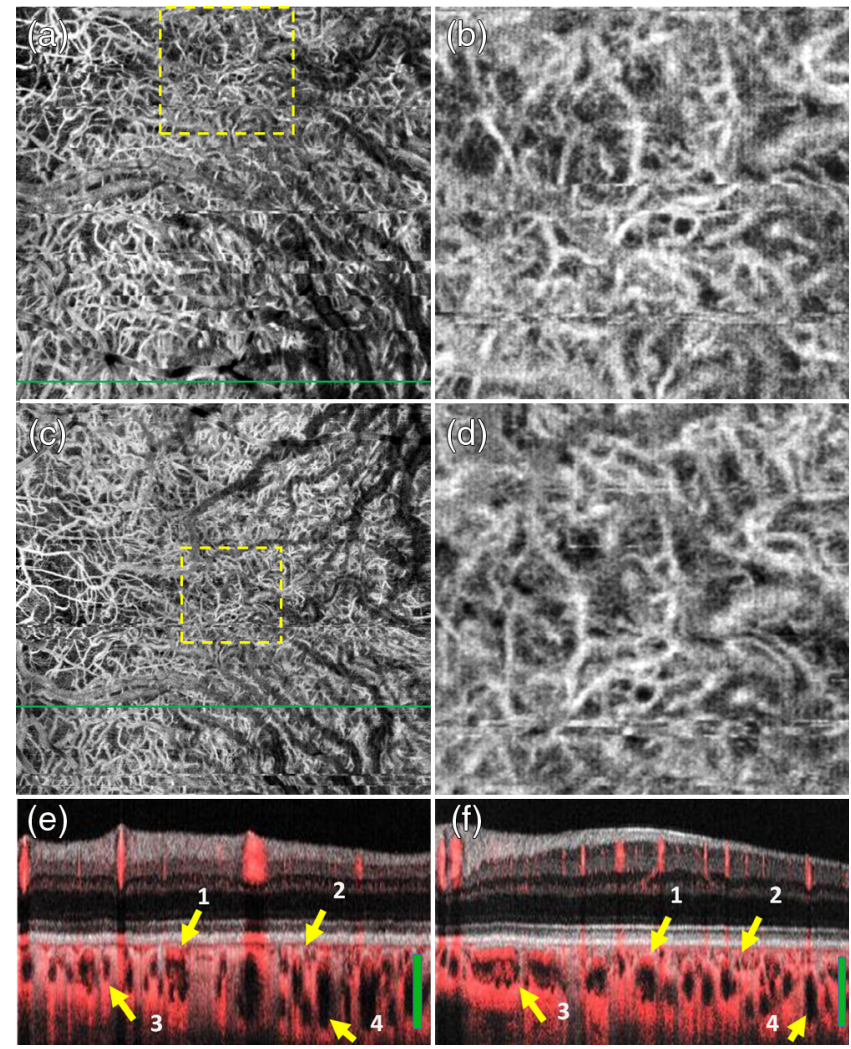

Fig. 7 FDML SSOCT at 1.7-MHz A-scan rate, PV OCTA en face projections of the choroid. Normal subject, age 51. Left column: scanning step $5 \mu \mathrm{m}$. Right column: scanning step $8 \mu \mathrm{m}$. Top row: full imaged area. Middle row: zoom at $1.5-\mathrm{mm} \times 1.5-\mathrm{mm}$ area centered at the fovea. Bottom row: overlay of OCTA images (red) and intensity Bscans (gray scale); arrows indicate example: 1 -large vessels with OCTA signal visible in the top part of the vessel, 2-small vessels with OCTA signal visible across the lumen, 3-vessels appearing as white in the en face projection due to inclusion of flow projections, and 4-vessels appearing as dark; green bars indicate position of the en face projections. Green line in en face angiograms (a) and (c) showing the location of the B-scans (e) and (f), respectively. Image sizes: (a) $5 \mathrm{~mm} \times 5 \mathrm{~mm}$, (b) $9 \mathrm{~mm} \times 9 \mathrm{~mm}$, and (c and d) $1.5 \mathrm{~mm} \times 1.5 \mathrm{~mm}$.

In Fig. 8, large choroidal vessels are visible as areas of low OCTA signal embedded in high OCTA noise (pixels with random, high OCTA signal values), and appear similar to shadows of choroidal vessels observed in the OCT intensity projections. This suggests that even though blood flow signal cannot be detected in large choroidal vessels with the commercial apparatus, some information about the architecture of the choroidal vasculature can be retrieved in the form of such shadowgrams [Figs. 8(e) and 8(f)].

\subsection{Comparison of High-Speed OCTA with FA and ICGA of a Normal Eye}

To preserve imaging resolution, the spot diameter of the imaging beam should not exceed half of the $1 / e^{2}$ beam diameter at the retina. Large scan steps cause loss of information about object features of high spatial frequencies (e.g., choriocapillaris), which could be obtained from higher scanning densities. This, however, compromises the capability of wide-field imaging with OCT angiography. Acquisition of data from only 35-deg FOV, considered as "standard" rather than "wide" in ophthalmic diagnostics, 

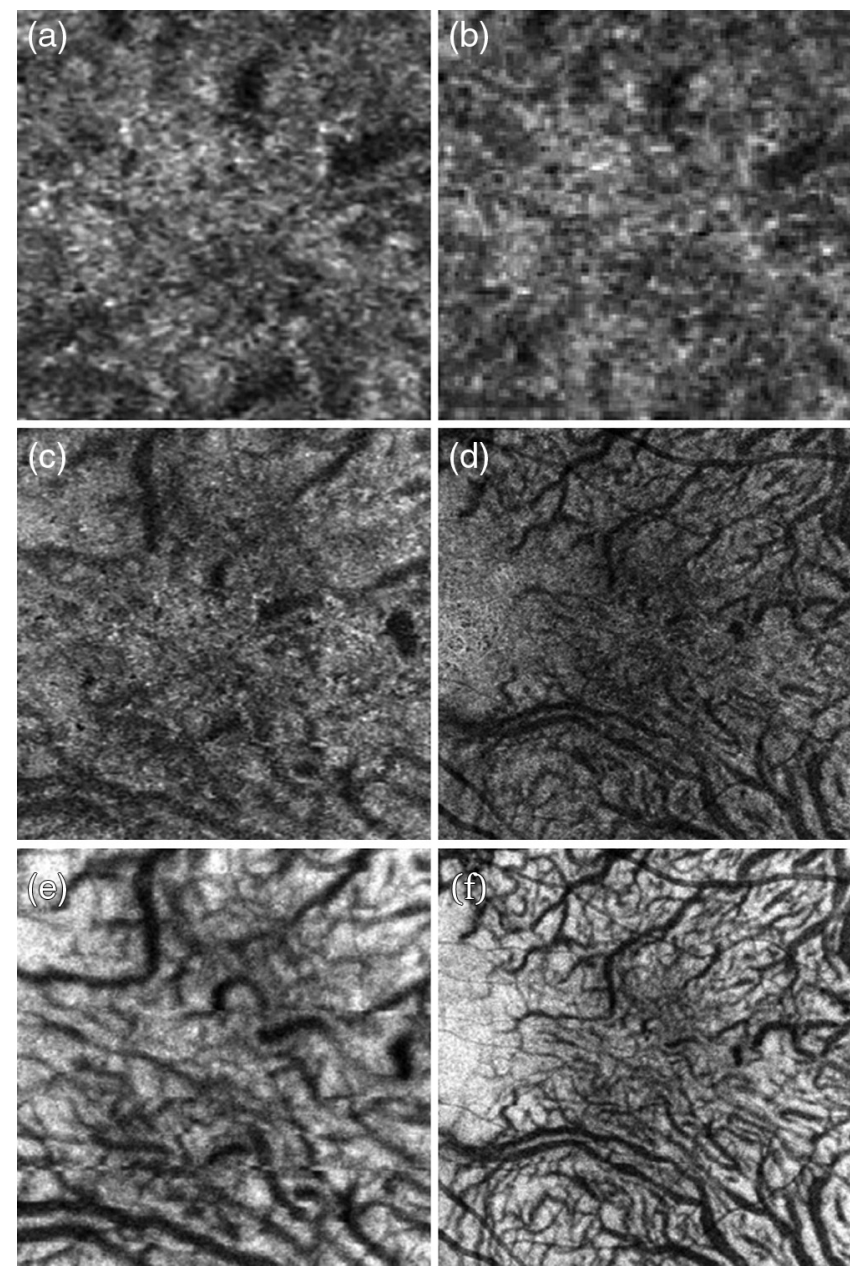

Fig. 8 Zeiss Angioplex 68-kHz, A-scan rate, and OCTA and OCT intensity en face projections of the choroid. Normal subject, age 51. Left column: scanning step $12 \mu \mathrm{m}$. Right column: scanning step $17 \mu \mathrm{m}$. Top row: OCTA, zoom at $1.5-\mathrm{mm} \times 1.5-\mathrm{mm}$ area centered at the fovea. Middle row: OCTA, full imaged area. Bottom row: OCT intensity projections. Image sizes: $(a$ and b) $1.5 \mathrm{~mm} \times 1.5 \mathrm{~mm}$, (c and e) $3 \mathrm{~mm} \times 3 \mathrm{~mm}$, and ( $\mathrm{d}$ and f) $6 \mathrm{~mm} \times 6 \mathrm{~mm}$. would take an unreasonably long time. A common solution to this challenge is breaking the OCTA imaging into acquisitions of data from smaller areas in neighboring locations in the object, and numerically "stitching" the resultant images into mosaics.

In Figs. 9-11, we demonstrate the results of stitching images from 43 -D datasets, each covering an area of $5 \times 5 \mathrm{~mm}$ of the retina. The resultant mosaics span 30 -deg FOV, enabling sideby-side comparison with corresponding images obtained using standard ophthalmic diagnostic methods.

Figure 9 compares a color fundus photograph with an OCT intensity projection image obtained from the healthy volunteer (Caucasian female, age: 51 years). The mosaic generated from full retinal depth projections [Fig. 9(b)] shows a network of large vessels, with structural details similar to FP [Fig. 9(a)]. However, it lacks the color discrimination of features provided by the fundus photos, which enables differentiation between large arteries and veins. Although this OCT image was generated from data with B-scan repeats to enable OCT angiography computations, data acquisition replications are not required in the OCT intensity projection imaging. ${ }^{37}$ Capturing OCT intensity data spanning a 30-deg FOV, without exceeding the half-ofthe- $1 / e^{2}$ beam spot diameter step, would take only $1 \mathrm{~s}$ with $1.7-$ $\mathrm{MHz}$ imaging rate. Imaging a 60-deg FOV would take $3.8 \mathrm{~s}$. However, only limited information about retinal vascular structures is available, e.g., retinal capillaries are not visualized in full depth intensity projections, and can be visualized only in projections from segmented selected retinal layers. ${ }^{37,39}$ To reiterate, 1.7-MHz imaging rate could be utilized for high-definition (densely sampled), wide field, intensity OCT imaging in single datasets but only at the cost of losing vascular imaging capability and imaging sensitivity.

Figure 10 enables comparison of retinal vascular imaging with FA and OCT angiography at $1.7-\mathrm{MHz}$ A-scan rate. OCT angiography has an advantage over the FA in imaging of the architecture of capillary vessels. Selected regions of interest of the 30-deg FOV mosaic obtained from the FDML SSOCT system can be enlarged to analyze the details of retinal capillaries [Figs. 10(c) and 10(d)], which are not visible in FA. However, FA can provide information about time dynamics of blood distribution in different vessels, which is not available with OCTA. Also, OCTA imaging relies on the motion contrast, i.e., OCTA signal can be detected from the flow of highly scattering blood
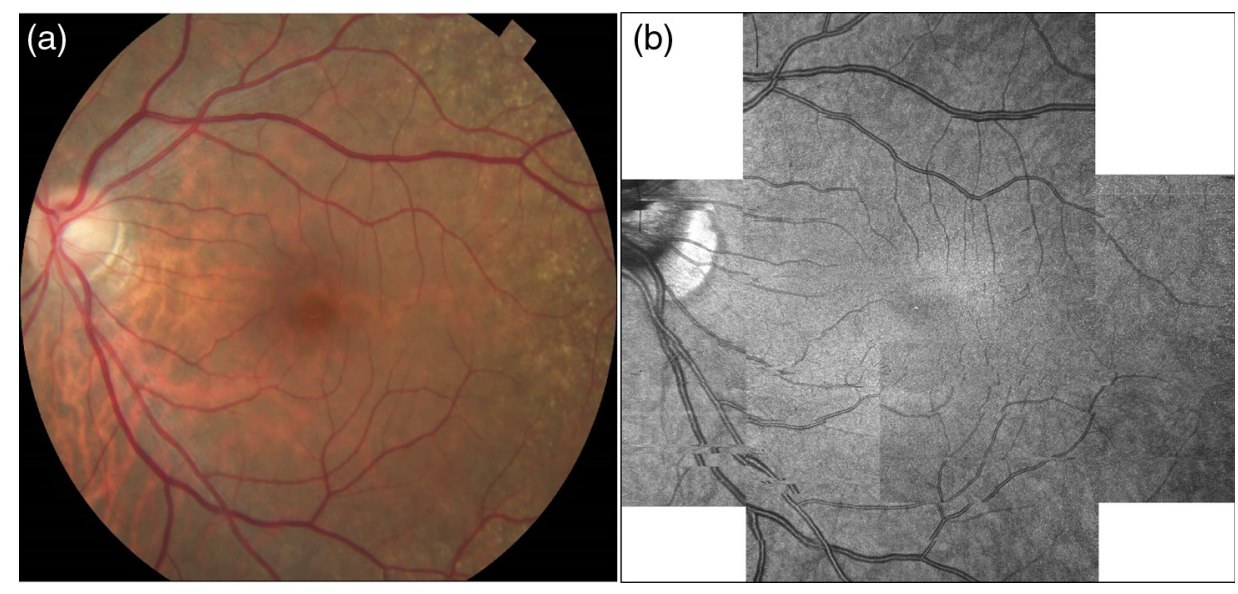

Fig. 9 Comparison of fundus photograph (30-deg FOV) with OCT intensity full-depth projection, 30-deg mosaic composed of four 5- $\mathrm{mm}^{2}$ OCT images. Images obtained in the volunteer N1-Caucasian female, 51-years-old. 

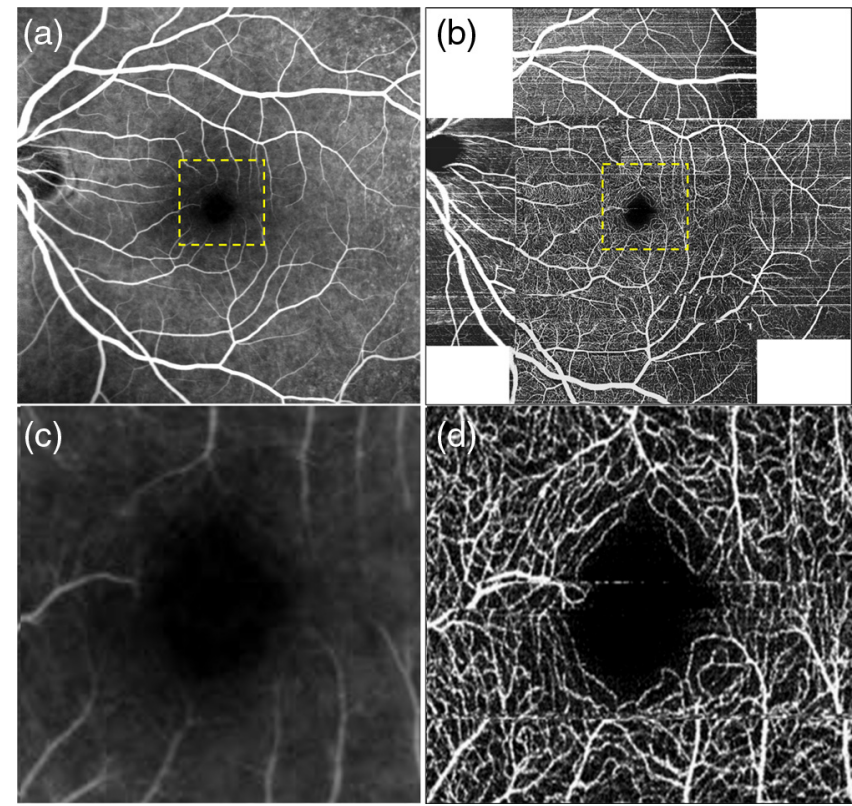

Fig. 10 Comparison of FA, 3 min after fluorescein injection (a, c) with PV OCTA en face projection of the retinal vasculature [(b and d) 30deg mosaic composed of four 5-mm $\times 5-\mathrm{mm}$ OCT images]. Right row: zoom at 1.5-mm $\times 1.5-\mathrm{mm}$ area centered at the fovea. Image sizes: (a and b) $9 \mathrm{~mm} \times 9 \mathrm{~mm}$ and (c and d) $1.5 \mathrm{~mm} \times 1.5 \mathrm{~mm}$.

cells, which is sufficiently fast to introduce measurable changes of the OCT signal. In consequence, leakage of blood or plasma from the vessels is not directly visualized in OCTA images.

Perhaps the most striking results were obtained in the imaging of the choroid (Fig. 11). The OCT intensity projection displayed in an inverted gray scale [Fig. 11(b)] mimics remarkably well the appearance of large choroidal vessels in ICGA $30 \mathrm{~s}$ after the injection of the fluorophore. The main difference between OCT angiography at 1.7-MHz A-scan rate and standard clinical diagnostic methods can be assessed from Figs. 11(a), 11(c), and 11(d). The 30-deg FOV OCTA mosaics reveal features not visualized in the ICG angiography. The ICGA not only misses the meshwork of capillary vessels [Fig. 11(c)] but also has limited capability to visualize an extremely rich network of highly convoluted medium-sized vessels [Fig. 11(d)]. Since the OCTA mosaics were generated from datasets acquired with high scanning density, regions of interest can be enlarged at any location of the 30-deg FOV to reveal highly detailed vascular features. The similarity between ICGA and OCTA is in the intrusion of retinal vessels in visualization of the choroid. In ICGA, the visibility of retinal vessels is caused by filling of the retinal vasculature with the fluorophore. In OCTA, the visibility of retinal vasculature in the projections of the choroid is caused by retinal blood flow projections. ${ }^{36}$

Eye motion is one of the main challenges in wide-field OCTA imaging. The imaging time required to collect each of the datasets covering $5 \mathrm{~mm} \times 5 \mathrm{~mm}$ area of the eye fundus with the FDML SSOCT system was $6 \mathrm{~s}$. Although there is no apparent degradation of the image quality related to possible drying of the tear film during the extended imaging time, motion artifacts are visible in all images. Artifacts connected to involuntary saccadic motion of the eye manifest as discontinuities in vessels, often accompanied by horizontal lines of high OCT angiography signal intensity [examples indicated by arrows in Fig. 10(b)].

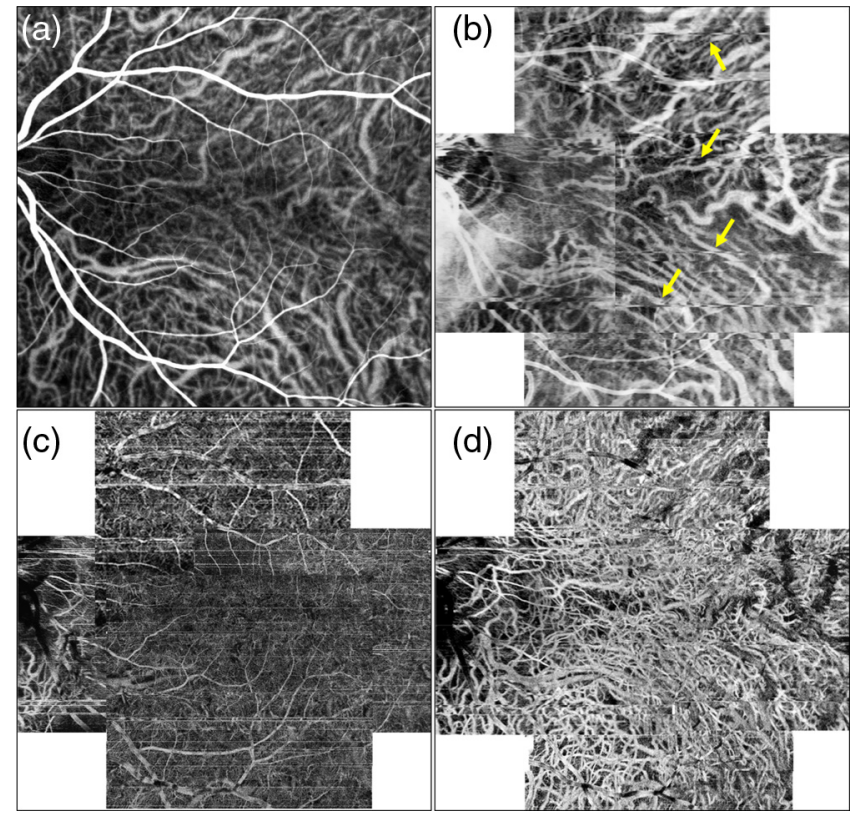

Fig. 11 Comparison of ICG angiography, (a) $30 \mathrm{~s}$ after the fluorophore injection with (b) OCT intensity projection of the choroid and (c) PV OCTA en face projection of the choriocapillaris, and (d) deeper choroid. OCT projections are mosaic composed of four $5-\mathrm{mm} \times 5-\mathrm{mm}$ OCT images spanning 30-deg FOV.

\subsection{Demonstration of 1.7-MHz A-Scan Rate OCT Angiography Imaging in Advanced AMD}

Imaging of eyes with developing pathology provides additional challenges in comparison with imaging of normal subjects. Figures 12-15 show three cases of advanced AMD. Patient P1, age 60 years, Caucasian male (Fig. 12), was diagnosed with extensive large drusen [green arrow in Fig. 12(b)], central GA, and juxtafoveal lobules of GA (yellow arrow). He had good transparency of ocular media and good eye stability, which enabled imaging of the retinal and choroidal vasculature with contrast similar to that of a normal eye. A rich network of fine retinal capillary vessels is visible throughout the entire 30-deg FOV [Fig. 12(c)], with the 8- $\mu$ m scan step. In the OCTA en face projection of the choroid, a dense network of normal appearing large choroidal vessels is visible in the RPE atrophy areas. Choroidal vessels were not visualized outside the GA with an exception of sparse areas where their visibility is poor [red arrow in Fig. 12(d)]. This is most likely due to the presence of large confluent drusen, covering extensive areas at the border and outside the GA, and causing attenuation of light penetrating toward the choroid. Similar effect of low OCTA signal from the choroid, coinciding with drusen can be observed in Fig. 14(d). For comparison, a 25-deg FOV mosaic composed of three $5 \mathrm{~mm} \times 5 \mathrm{~mm}$ PV OCTA en face projections is presented in Fig. 13. Although the scan step was smaller $(5 \mu \mathrm{m})$, no new findings were revealed in comparison to Fig. 12. The retinal vasculature [Fig. 12(a)] shows a similarly detailed network of vessels down to the capillary level. The choroidal vasculature has a similar appearance to Fig. 12(d). This observation may suggest that in advanced stages of disease development, with large-scale pathological changes, the advantages of a large FOV may surpass the need for high detail imaging.

Patient P2 (age 67 years, Caucasian female) had exudative macular degeneration treated with anti-vascular endothelial 

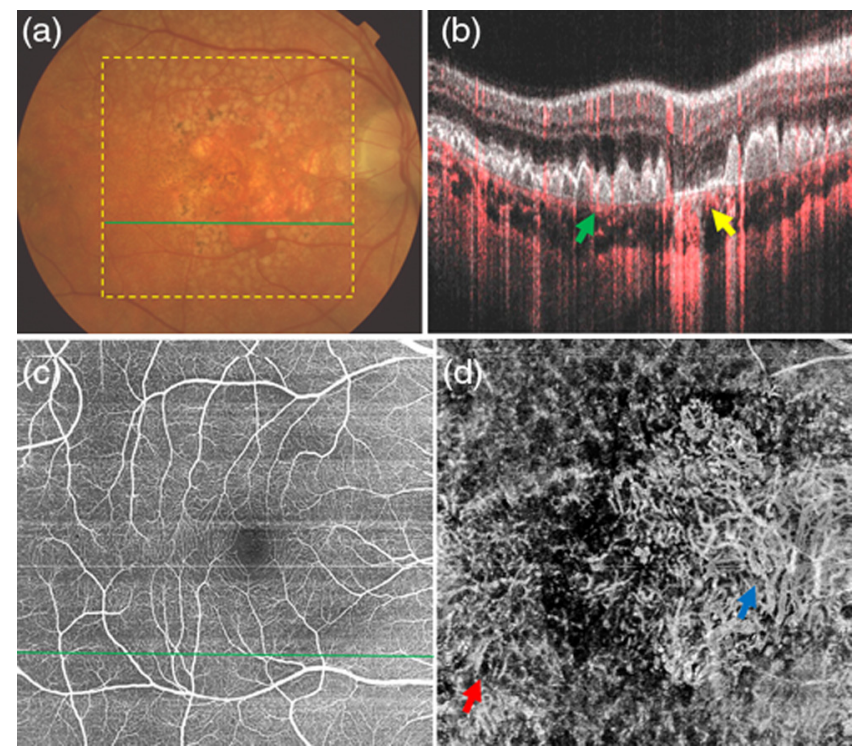

Fig. 12 Patient $\mathrm{P} 1,60$-years Caucasian male diagnosed with geographic atrophy. (a) Fundus photograph and (b) OCT (gray scale) and OCTA (red scale) composite B-scan image; green arrow indicates large drusen, yellow arrow points at the RPE atrophy. (c) En face projection of the retinal vasculature. (d) OCTA projection of combined Sattler's layer and Haller's layer vessels; blue arrow indicates vessels in GA area, red arrow points vessels outside GA area. Green line in (a) and en face angiograms (c) showing the location of the Bscans (b). OCT image sizes: $9 \mathrm{~mm} \times 9 \mathrm{~mm}$ (30-deg FOV).

growth factor (anti-VEGF) injections (4 times) (Fig. 14). The area of RPE damage was smaller than in patient P1 with GA. However, the OCT imaging sensitivity was lower than in P1, most likely due to poorer transparency of the ocular media, resulting in low visibility of the inner retinal layers [Fig. 14(b)]. In addition, due to lower eye stability during the imaging, motion artifacts have higher influence on the image quality than in $\mathrm{P} 1$.

The OCTA en face projection of the choroid [Fig. 14(d)] visualizes large vessels throughout the entire image. However, the vessels are less sharply defined than in a normal eye except in the area of GA, where clear visualization of large vessels was achieved. In addition, focal areas of low OCTA signal are visible in the choroidal en face projection, which coincides with the location of drusen in the fundus photograph [arrows in Figs. 14(a) and 14(d)].

Patient P3, an 81-year-old Caucasian male with nonexudative AMD, with geographic atrophy and cataract, had the most advanced pathology of the presented cases. A very extensive GA area spanned the majority of the macular region of the retina. Poor transparency of the ocular media degraded the sensitivity of OCT imaging resulting in poor visualization of the inner retinal layers [Fig. 15(b)] and, in consequence, also in poor visualization of the retinal vasculature in the OCTA en face projection [Fig. 15(c)]. The choroidal vessels are visible in the entire imaged area [Fig. 15(d)]; however, their appearance is diffuse outside the GA areas. In the GA areas, the vessels are sharply defined but notably, their density appears to be much lower than in the GA area in patient P1 [Fig. 12(d)] and in the normal eye [Figs. 7 and 11(c)]. This may suggest loss of deeper vessels due to the development of pathology.

The choriocapillaris layer was not visualized in any of the en face projections of the three presented patients. Two possible explanations are choriocapillaris atrophy, and errors in image segmentation and flattening. The composite OCT-OCTA crosssectional images reveal only sparse spots of OCTA signal within a narrow layer abutting the Bruch's membrane in P1 [Fig. 12(b)], whereas a corresponding layer of the normal eye is more densely occupied by choriocapillaris [Fig. 12(b)]. In patients $\mathrm{P} 2$ and $\mathrm{P} 3$, choriocapillaris is nearly absent in the cross-sectional images [Figs. 14(b) and 15(b)]. This observation may support the hypothesis of choriocapillaris atrophy. However, the image segmentation and flattening errors cannot be ruled out either. Geographic atrophy is perhaps one of the most challenging pathologies for data processing algorithms aiming at segmentation of the RPE. Not only is the RPE missing in large areas of the retina but also large drusen disrupting the laminar appearance of the outer retinal layers are often present adjacent to GA. As a result, data segmentation algorithms often fail in correct identification of the RPE location and in approximation of the normal curvature of the outer retina, which serves as a reference for the en face projection image generation. Segmentation errors are present in all presented cases affecting the visualization of choriocapillaris and deeper choroid. Image flattening errors are
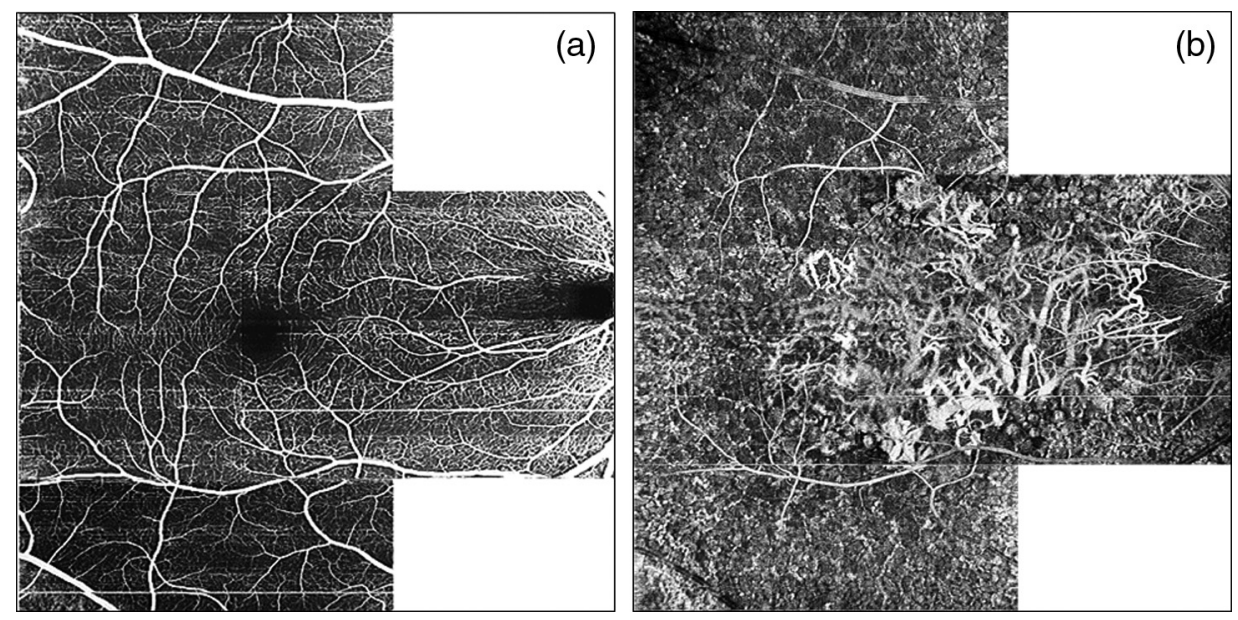

Fig. 13 Patient P1, 60-year-old Caucasian male, PV OCTA en face projections of (a) the retinal and (b) choroidal vasculature. Mosaics were generated from three datasets covering $5 \mathrm{~mm} \times 5 \mathrm{~mm}$, each. Image sizes: $\sim 9 \mathrm{~mm} \times 6 \mathrm{~mm}(25-\times 18$ - deg FOV). 


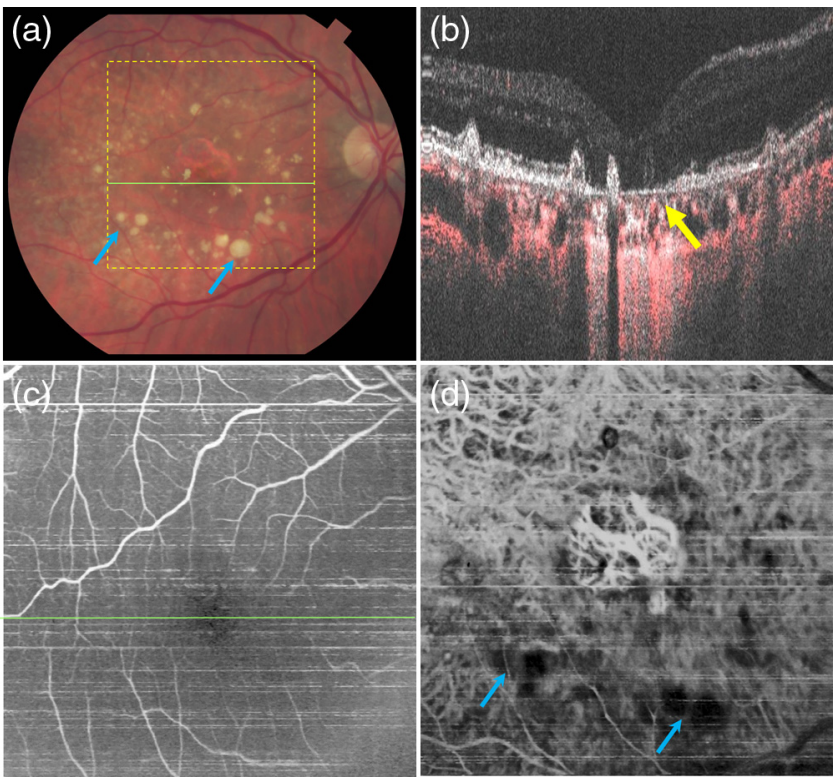

Fig. 14 Patient P2, 67-year-old Caucasian female, exudative AMD treated with anti-VEGF injections. (a) Color fundus photograph (35deg FOV). (b) OCT (gray scale) and OCTA (red scale) composite B-scan image with yellow arrow points at the RPE atrophy. (c) en face projection view of retinal layers. (d) OCTA projections of combined Sattler's layer and Haller's layer vessels, blue arrows point at the low signal areas corresponding to drusen in (a). Green line in (a) and en face angiograms (c) showing the location of the Bscans (b). OCT image sizes: $5 \times 5 \mathrm{~mm}$ (16-deg FOV).

visible in the right bottom corner of Fig. 14(c) (diminished visibility of retinal vessels) and in the right edge of Fig. 15(c) (intrusion of the choroidal vasculature in the retinal en face projection image visible as a band of high OCTA signal).

\section{Discussion and Conclusions}

In this study, we have used an FDML SSOCT system with imaging speed of 1.7 million A-scans/s to demonstrate advantages and challenges of "wide-field" OCTA imaging of the posterior segment of the human eye. In comparison with previous reports on wide-field OCTA imaging (Table 6), we have implemented scan protocols with equal scanning density in the horizontal and vertical directions, with scan steps of $\sim 0.4$ and $\sim 0.6$ of the $1 / e^{2}$ beam spot diameter at the retina (5 and $8 \mu \mathrm{m}$, respectively). We have demonstrated that scanning steps smaller than half of the $1 / e^{2}$ beam spot diameter enable visualization of vascular features down to the capillary level and are important in visualization of choriocapillaris layer morphology. However, they limit the FOV of the data acquired within a time limit of $6 \mathrm{~s}$ to $16 \mathrm{deg}$ $(5 \mathrm{~mm} \times 5 \mathrm{~mm})$. Imaging with scan steps exceeding half of $1 / e^{2}$ beam spot diameter $(8 \mu \mathrm{m})$ enabled widening the FOV captured in a single dataset to $30 \mathrm{deg}$. Good rendering of retinal capillaries was still achieved at this scanning density but visualization of choriocapillaris architecture had lower apparent contrast. In comparison, slower imaging systems ( 70 to $100 \mathrm{kHz})$ undersample the scanned area (scan step larger than the $1 / e^{2}$ beam spot diameter), yet still cannot achieve the standard $\sim 35$ - deg FOV offered by the classic fundus angiography (Table 6). We demonstrated that in a commercial OCT system operating at a $68-\mathrm{kHz}$ A-scan rate, with 10-deg FOV, scanning steps comparable to the beam spot diameter provide details of the retinal vasculature similar to features visualized with the FDML SSOCT system at $1.7-\mathrm{MHz}$ imaging rate with $30-\mathrm{deg}$

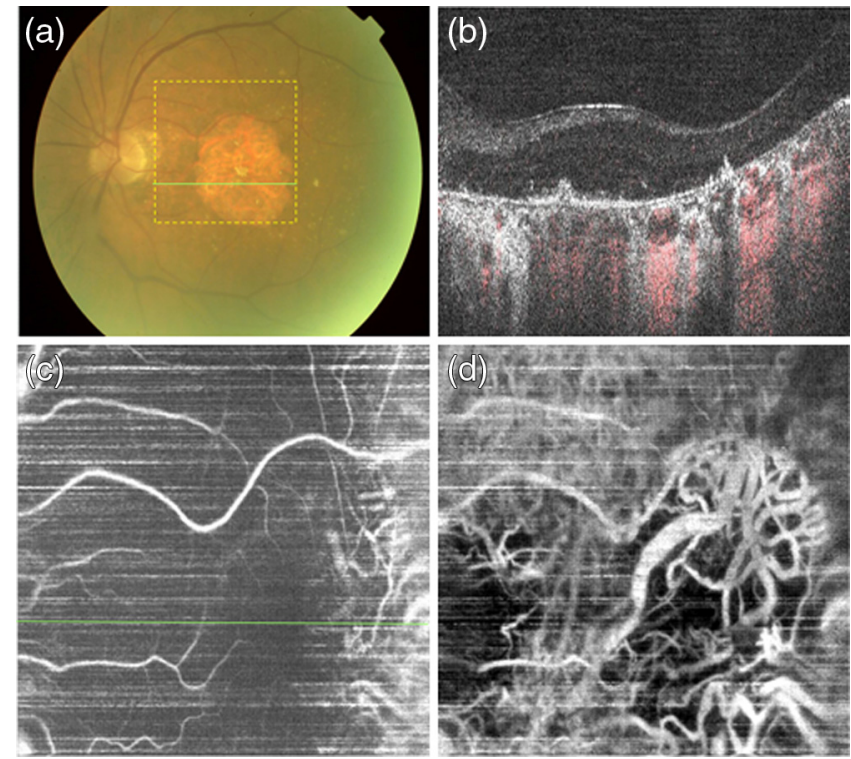

Fig. 15 Patient P3, 81-year-old Caucasian male with nonexudative AMD, with geographic atrophy and cataract. (a) Color fundus photograph, 35-deg FOV. (b) OCT (gray scale) and OCTA (red scale) composite B-scan. (c) En face projection view of retinal layers. (d) OCTA projections of combined Sattler's layer and Haller's layer vessels. Green line in (a) and en face angiograms (c) showing the location of the B-scans (b). OCT image sizes: $5 \mathrm{~mm} \times 5 \mathrm{~mm}$ (16-deg FOV).

FOV. However, undersampling (17- $\mu \mathrm{m}$ scanning step, 20-deg FOV) resulted in loss of information about the retinal capillary network. This observation is consistent with a previous report on wide-field FDML SSOCTA imaging at $1.7-\mathrm{MHz}$ A-scan rate ${ }^{38}$ in which 48-deg FOV, i.e., nearly the FOV of a classic wide-field FA, was achieved by exceeding the half beam spot diameter step size in the fast scan axis, and undersampling in the slow scan axis (Table 6). The authors reported visualization of large retinal vessels but did not achieve visualization of the retinal capillaries nor choriocapillaris morphology. Only narrowing the FOV to 12 deg revealed the retinal capillary system and "gaps" (flow voids) in the OCTA signal from the choroid, characteristic of the choriocapillaris layer.

OCT angiography has been shown to provide detailed, depthresolved images of vascular structures of the retina and choroid down to the capillary level. However, the challenge is in achieving a large FOV within imaging times not exceeding 5 to $10 \mathrm{~s}$. A common solution to enable wide FOV without sacrificing the visualization of fine vascular features is implementation of mosaic-like imaging of the eye fundus. We have demonstrated that with $1.7-\mathrm{MHz}$ imaging rate a $30-\mathrm{deg}$ FOV can be visualized with only four datasets acquired at neighboring locations in the retina. We have compared the OCTA en face projections with "standard FOV" ( 35 to $40 \mathrm{deg}$ ) ophthalmic imaging. The OCT counterpart of FP is intensity (or structural) OCT imaging. It has the potential to achieve wide FOV as defined by clinical diagnostic standards, since it does not require repetitive acquisition of data from the same location in the eye fundus, essential in OCT angiography. However, only limited information about the vascular structures of the eye can be obtained from structural OCT imaging and no color information is available. Information corresponding to FA and ICGA can be obtained from OCT angiography imaging. OCTA can provide images of fine retinal vascular structures typically not observed in FA imaging. OCT imaging of the retinal vascular architecture was available since 
Table 6 Comparison between different OCT angiography systems.

\begin{tabular}{|c|c|c|c|c|c|c|}
\hline & & & & & Angiovue $\mathrm{T}^{\mathrm{TM}}$ & \\
\hline & Curren & t paper & Blatter et al. ${ }^{38}$ & Wang et al. ${ }^{44}$ & Optovue ${ }^{43}$ & Zeiss AngioPlex ${ }^{45,48}$ \\
\hline Parameters & & & & & & \\
\hline A-scan rate & $1.7 \mathrm{MHz}$ & $1.7 \mathrm{MHz}$ & $1.7 \mathrm{MHz}$ & $100 \mathrm{kHz}$ & $70 \mathrm{kHz}$ & $68 \mathrm{kHz}$ \\
\hline Scanning area & $5 \times 5 \mathrm{~mm}^{2}$ & $9 \times 9 \mathrm{~mm}^{2}$ & $16 \times 16 \mathrm{~mm}^{2}$ & $9 \times 9 \mathrm{~mm}^{2}, 12 \times 12 \mathrm{~mm}^{2}$ & $6 \times 6 \mathrm{~mm}^{2}, 8 \times 8 \mathrm{~mm}^{2}$ & $6 \times 6 \mathrm{~mm}^{2}$ \\
\hline B-scan repeats & 5 & 5 & 5 & 2 & 2 & 2 \\
\hline Number of B-scans & 1024 & 1152 & 800 & 500 & 304 & 350 \\
\hline $\begin{array}{l}\text { Number of A-scans } \\
\text { per B-scan }\end{array}$ & 1024 & 1152 & 2060 & 500 & 304 & 350 \\
\hline Beam spot diameter $(\mu \mathrm{m})$ & 14 & 14 & 14 & 15 & 15 & 15 \\
\hline Horizontal step size & $5 \mu \mathrm{m}$ & $8 \mu \mathrm{m}$ & $8 \mu \mathrm{m}$ & $18 \mu \mathrm{m}$ for $9 \times 9 \mathrm{~mm}^{2}$ & $20 \mu \mathrm{m}$ for $6 \times 6 \mathrm{~mm}^{2}$ & $17 \mu \mathrm{m}$ \\
\hline Vertical step size & $5 \mu \mathrm{m}$ & $8 \mu \mathrm{m}$ & $20 \mu \mathrm{m}$ & $18 \mu \mathrm{m}$ for $9 \times 9 \mathrm{~mm}^{2}$ & $20 \mu \mathrm{m}$ for $6 \times 6 \mathrm{~mm}^{2}$ & $17 \mu \mathrm{m}$ \\
\hline $\begin{array}{l}\text { B-scan acquisition } \\
\text { time }(\mathrm{ms})\end{array}$ & 1.2 & 1.35 & 1.12 & 5 & 5 & 5 \\
\hline $\begin{array}{l}\text { Volume acquisition } \\
\text { time (s) }\end{array}$ & 6 & 8 & 7 & 5 & 5 & 5 \\
\hline $\begin{array}{l}\text { Line scan ophthalmoscope } \\
\text { or eye tracking }\end{array}$ & No & No & No & Yes & Yes & Yes \\
\hline
\end{tabular}

the advent of the OCTA techniques. However, only high-speed systems at $\sim 1050$-nm wavelength imaging regimes are currently capable of visualization of the choroidal blood flow. We have demonstrated unprecedented detail and richness of choroidal vascular architecture imaged in a normal human eye imaged at $1.7-\mathrm{MHz} \mathrm{A}$-scan rate.

In addition, comparison between the OCT and classic angiographic imaging of the eye highlights the speed challenge in wide-field OCTA, not present in FP and angiography. Acquisition of OCTA datasets enabling visualization of retinal capillaries and choriocapillaris throughout a modest 16-deg FOV takes $6 \mathrm{~s}$ at 1.7-MHz imaging rate. During that time, involuntary eye motion may introduce artifacts obscuring the images of the posterior eye segment vasculature, including discontinuities of vessels caused by saccadic motion and distortions of the vascular networks caused by eye drifts. The severity of image deformation depends on the individual subject's eye stability during the OCT scanning. As demonstrated in the imaging of geographic atrophy cases, degradation of image quality caused by motion artifacts can be negligible (patient P1) or may substantially degrade the quality of images (patient P3). This observation indicates the need for development of experimental and numerical motion artifact compensation methods for OCT angiography, especially when wide-field imaging is considered.

Imaging of geographic atrophy cases revealed yet another challenge associated with depth-resolved OCTA visualization of vascular layers. With increasing severity of outer retinal pathology disrupting the laminar arrangement of the retinal layers and the integrity of the RPE, the numerical image segmentation and flattening methods are more susceptible to errors. Such errors may cause generation of incomplete or fragmented en face projection images of vascular networks, especially confined to narrow depth ranges (e.g., choriocapillaris). Caution is advised when interpreting the OCTA projections if there is a suspicion of failure in the image segmentation algorithm.

As a final remark, we address a common misconception connected to high-speed OCT angiography imaging. Increasing the imaging speed in OCT systems with beam scanning does not allow imaging time to be shortened to the point that no eye motion occurs during the data acquisition. Aside from the consideration of the imaging sensitivity diminishing with increasing imaging speed, the dynamics of the blood flow needs to be taken into account when developing experimental OCTA imaging protocols. OCT angiography relies on the detection of signal fluctuations (variation of magnitude and/or phase of the complex amplitudes) originating from moving blood cells. Therefore, the same locations of the eye fundus must be imaged repeatedly over a time period sufficiently long to enable observable signal change (i.e., change of the magnitude and/or phase of the complex amplitude larger than amplitude and/or phase noise levels). The length of this time period depends on the blood flow velocity in the vascular systems intended for visualization in OCTA angiograms. The slower the flow, the longer the time period of the repeated scanning required to detect signal change caused by moving blood cells and to visualize the location of vessels, at given imaging sensitivity and noise levels. Thus, the time limits in OCT angiography imaging are set not only by the speed of state-of-the-art OCT systems, but also by the dynamics of the ocular blood circulation.

\section{Disclosures}

The authors have no relevant financial interests in this article and no potential conflicts of interest to disclose. 


\section{Acknowledgments}

We gratefully acknowledge the contributions of VSRI UC Davis lab members especially, Susan Garcia (VSRI, UC Davis Medical Center) in subject recruitment, as well as Thomas Klein and Wolfgang Weiser (OptoRes $\mathrm{GmbH}$ ) for technical advice on the FDML laser. This research was supported by the National Eye Institute (R01EY024239), Research to Prevent Blindness and the Larry L. Hillblom Foundation.

\section{References}

1. J. T. Durham et al., "Microvascular modifications in diabetic retinopathy," Curr. Diabetes Rep. 11, 253-264 (2011).

2. Y. Imamura et al., "Enhanced depth imaging optical coherence tomography of the choroid in central serous chorioretinopathy," Retina $\mathbf{2 9}$, 1469-1473 (2009).

3. R. Ehrlich et al., "Age-related ocular vascular changes," Graefes Arch. Clin. Exp. Ophthalmol. 247, 583-591 (2009).

4. A. P. Cherecheanu et al., "Ocular perfusion pressure and ocular blood flow in glaucoma," Curr. Opin. Pharmacol. 13, 36-42 (2013).

5. S. S. Shoughy et al., "Update on wide- and ultra-widefield retinal imaging," Indian J. Ophthalmol. 63(7), 575-581 (2015).

6. M. T. Witmer et al., "Comparison of ultra-widefield fluorescein angiography with the Heidelberg 7. Spectralis $\left({ }^{\circledR}\right)$ noncontact ultra-widefield module versus the Optos $\left({ }^{\circledR}\right)$ and Optomap $\left({ }^{\circledR}\right), "$ Clin. Ophthalmol. 7, 389-94 (2013).

7. G. Staurenghi et al., "Scanning laser ophthalmoscopy and angiography with a wide-field contact lens system," Arch. Ophthalmol. 123(2), 244252 (2005).

8. B. Potsaid et al., "Ultrahigh speed spectral/Fourier domain OCT ophthalmic imaging at 70,000 to 312,500 axial scans per second," Opt. Express 16(19), 15149-15169 (2008).

9. L. An et al., "High speed spectral domain optical coherence tomography for retinal imaging at 500,000 A-lines per second," Biomed. Opt. Express 2(10), 2770-2783 (2011).

10. O. P. Kocaoglu et al., "Adaptive optics optical coherence tomography at $1 \mathrm{MHz}$," Biomed. Opt. Express 5(12), 4186-4200 (2014).

11. B. Potsaid et al., "Ultrahigh speed $1050 \mathrm{~nm}$ swept source/Fourier domain OCT retinal and anterior segment imaging at 100,000 to 400,000 axial scans per second," Opt. Express 18, 20029-20048 (2010).

12. B. Braaf et al., "Phase-stabilized optical frequency domain imaging at $1-\mu \mathrm{m}$ for the measurement of blood flow in the human choroid," Opt. Express 19(21), 20886-20903 (2011).

13. I. Grulkowski et al., "Retinal, anterior segment and full eye imaging using ultrahigh speed swept source OCT with vertical-cavity surface emitting lasers," Biomed. Opt. Express 3, 2733-2751 (2012).

14. Y. J. Hong et al., "High-penetration swept source Doppler optical coherence angiography by fully numerical phase stabilization," Opt. Express 20(3), 2740-2760 (2012).

15. W. J. Choi et al., "Phase-sensitive swept-source optical coherence tomography imaging of the human retina with a vertical cavity surface-emitting laser light source," Opt. Lett. 38(3), 338-340 (2013).

16. C. D. Lu et al., "Handheld ultrahigh speed swept source optical coherence tomography instrument using a MEMS scanning mirror," Biomed. Opt. Express 5, 293-311 (2014).

17. R. Poddar et al., "In vivo imaging of human vasculature in the chorioretinal complex using phase-variance contrast method with phase-stabilized 1- $\mu \mathrm{m}$ swept-source optical coherence tomography," J. Biomed. Opt. 19(12), 126010 (2014).

18. T. Klein et al., "High-speed OCT light sources and systems," Biomed. Opt. Express 8(2), 828-860 (2017).

19. T. Klein et al., "Megahertz OCT for ultrawide-field retinal imaging with a $1050 \mathrm{~nm}$ Fourier domain mode-locked laser," Opt. Express 19(4), 3044-3062 (2011).

20. T. Bonin et al., "In vivo Fourier-domain full-field OCT of the human retina with 1.5 million A-lines/s," Opt. Lett. 35(12), 3432-3434 (2010).

21. R. A. Leitgeb et al., "Doppler optical coherence tomography," Prog. Retinal Eye Res. 41, 26-43 (2014).

22. H. Ren et al., "Imaging and quantifying transverse flow velocity with the Doppler bandwidth in a phase-resolved functional optical coherence tomography," Opt. Lett. 27, 409-411 (2002).
23. S. G. Proskurin et al., "Determination of flow velocity vector based on Doppler shift and spectrum broadening with optical coherence tomography," Opt. Lett. 28, 1227-1229 (2003).

24. N. Uribe-Patarroyo et al., "Quantitative technique for robust and noisetolerant speed measurements based on speckle decorrelation in optical coherence tomography," Opt. Express 22, 24411-24429 (2014).

25. S. Makita et al., "Optical coherence angiography," Opt. Express 14, 7821-7840 (2006).

26. Q. Wang et al., "Vascular density in retina and choriocapillaris as measured by optical coherence tomography angiography," Am. J. Ophthalmol. 168, 95-109 (2016).

27. K. Kurokawa et al., "Adaptive optics optical coherence tomography angiography for morphometric analysis of choriocapillaris," Biomed. Opt. Express 8(3), 1803-1822 (2017).

28. A. Mariampillai et al., "Speckle variance detection of microvasculature using swept-source optical coherence tomography," Opt. Lett. 33(13), 1530-1532 (2008).

29. J. Fingler et al., "Volumetric microvascular imaging of human retina using optical coherence tomography with a novel motion contrast technique," Opt. Express 17, 22190-22200 (2009).

30. Y. Jia et al., "Split-spectrum amplitude-decorrelation angiography with optical coherence tomography," Opt. Express 20(4), 4710-4725 (2012).

31. S. Makita et al., "Noise-immune complex correlation for optical coherence angiography based on standard and Jones matrix optical coherence tomography," Biomed. Opt. Express 7(4), 1525-1548 (2016).

32. R. K. Wang et al., "Three-dimensional optical angiography," Opt. Express 15, 4083-4097 (2007).

33. R. K. Wang et al., "Depth-resolved imaging of capillary networks in retina and choroid using ultrahigh sensitive optical microangiography," Opt. Lett. 35(9), 1467-1469 (2010).

34. H. Ren et al., "Power optical Doppler tomography imaging of blood vessels in human skin and M-mode Doppler imaging of blood flow in chick chrioallantoic membrane," Proc. SPIE 4956, 225 (2003).

35. E. K. Chin et al, "Staging of macular telangiectasia: power-Doppler optical coherence tomography and macular pigment optical density," Invest. Ophthalmol. Vis. Sci. 54, 4459-4470 (2013).

36. I. Gorczynska et al., "Comparison of amplitude-decorrelation, specklevariance and phase-variance OCT angiography methods for imaging the human retina and choroid," Biomed. Opt. Express 7(3), 911-942 (2016).

37. J. P. Campbell et al., "Detailed vascular anatomy of the human retina by projection-resolved optical coherence tomography angiography," Sci. Rep. 7, 42201 (2017).

38. C. Blatter et al., "Ultrahigh-speed non-invasive widefield angiography," J. Biomed. Opt. 17(7), 070505 (2012).

39. W. Choi et al., "Choriocapillaris and choroidal microvasculature imaging with ultrahigh speed OCT angiography," PLoS One 8(12), e81499 (2013).

40. D. Y. Kim et al., "Optical imaging of the chorioretinal vasculature in the living human eye," Proc. Natl. Acad. Sci. 110(35), 14354-14359 (2013).

41. I. Gorczynska et al., "Imaging of the human choroid with a $1.7 \mathrm{MHz}$ Ascan rate FDML swept source OCT system," Proc. SPIE 10045, 1004510 (2017).

42. Q. Q. Zhang et al., "Wide-field optical coherence tomography based microangiography for retinal imaging," Sci. Rep. 6, 22017 (2016).

43. Optovue, Inc., "Angiovue," http://www.optovue.com/products/angiovue/ (23 Feb 2017).

44. R. K. Wang et al., "Wide field OCT angiography enabled by 2 repeated measurements of B-scans," Opt. Lett. 41(10), 2330-2333 (2016).

45. Carl Zeiss Meditec AD, "ZEISS CIRRUS OCT with AngioPlex," https://www.zeiss.com/meditec/int/c/angioplex-oct-angiography.html (23 February 2017).

46. P. Zang et al., "Automated three-dimensional registration and volume rebuilding for wide-field angiographic and structural optical coherence tomography," J. Biomed. Opt. 22(2), 026001 (2017).

47. ANSI, American National Standard for the Safe Use of Lasers, ANSI Z136.1, ANSI ${ }^{\circledR}$ Z136.1-2014, Revision of ANSI Z136.1-2007, Laser Institute of America, Orlando, Florida (2014).

48. P. J. Rosenfeld et al., "ZEISS Angioplex ${ }^{\mathrm{TM}}$ spectral domain optical coherence tomography angiography: technical aspects," Dev. Ophthalmol. 56, 18-29 (2016).

49. P. Ossowski et al., "Differentiation of morphotic elements in human blood using optical coherence tomography and a microfluidic setup," Opt. Express 23(21), 27724-27738 (2015). 
50. J. Schindelin et al., "Fiji: an open-source platform for biological-image analysis," Nat. Methods 9(7), 676-682 (2012).

51. B. Braaf et al., "Angiography of the retina and the choroid with phaseresolved OCT using interval-optimized backstitched B-scans," Opt. Express 20(18), 20516-20534 (2012).

52. Y. Dodo et al., "Clinical relevance of reduced decorrelation signals in the diabetic inner choroid on optical coherence tomography angiography," Sci. Rep. 7, 5227 (2017).

Raju Poddar received his BSc degree in physics, MSc degree in biophysics, MTech degree in biotechnology, and his PhD in technology. $\mathrm{He}$ has completed his postdocs from NTU, Singapore, Tsukuba University, Japan, and the University of California, Davis, USA (also as visiting assistant professor). Currently, he is an assistant professor at the Department of Bioengineering, Birla Institute of Technology, Ranchi, India. His current research interests include optical coherence tomography, medical imaging, and optoelectronic systems.

Justin V. Migacz received his BA degree in electrical engineering from the University of Colorado at Boulder, and his MS degree in electrical and computer engineering from Duke University. He is a graduate student of biomedical engineering at the University of California at Davis. Currently, he is a member of the laboratory of Professor John Werner in the Department of Ophthalmology. His research interests include optical coherence tomography, specifically in the applications of retinal angiography and eye surgery.
Daniel M. Schwartz received his medical degree from the UCSF. He then completed a residency in Ophthalmology at the Wilmer Institute, Johns Hopkins Hospital. He received fellowship training in vitreoretinal surgery at the Medical College of Wisconsin. He has been on the faculty at UCSF in the Department of Ophthalmology since 1990, where he is currently professor of clinical ophthalmology and director of the retina service at the UCSF and the VAMC, San Francisco.

John S. Werner is a distinguished professor of Ophthalmology and Neurobiology at the University of California, Davis. His research is focused on retinal structure and function in normal aging and disease. He received his $\mathrm{PhD}$ from Brown University, and conducted postdoctoral research at the Institute for Perception-TNO in Soesterberg, The Netherlands. He was previously a professor at the University of Colorado, Boulder.

Iwona Gorczynska has a doctoral degree in physics received from Nicolaus Copernicus University, Poland. She completed scientific trainings at Massachusetts Institute of Technology, USA and Tufts, New England Eye Center, USA (postdoctoral position), and at the University of California Davis, USA (visiting assistant professor). Currently, she is an assistant professor at Nicolaus Copernicus University, Poland. She is working on research projects in the field of biomedical imaging and biophysics at the Laboratory of Applied Biophotonics. 